\title{
The Relationship between Enterprise Resource Planning, Total Quality Management, Organizational Excellence, and Organizational Performance-the Mediating Role of Total Quality Management and Organizational Excellence
}

\author{
Hassan Saleh Al-Dhaafri ${ }^{1,2}$, Rushami Zien Bin Yusoff ${ }^{3} \&$ Abdullah Kaid Al-Swidi $^{3}$ \\ ${ }^{1}$ Dubai Police, Dubai, United Arab Emirates \\ ${ }^{2}$ Othman Yeop Abdullah Graduate School of Business, College of Business, University Utara Malaysia, \\ Malaysia \\ ${ }^{3}$ College of Business, University Utara Malaysia, Sintok, Malaysia \\ Correspondence: Hassan Saleh Al-Dhaafri, Othman Yeop Abdullah Graduate School of Business, College of \\ Business, University Utara Malaysia, Malaysia. Tel: 971-50-882-8812. E-mail: hassan_saleh3@hotmail.com
}

Received: April 12, 2014 Accepted: May 19, 2014 Online Published: June 24, 2014

doi:10.5539/ass.v10n14p158 URL: http://dx.doi.org/10.5539/ass.v10n14p158

\begin{abstract}
Purpose: This study was set up to examine the mediating effect of TQM and organizational excellence between ERP and organizational performance.

Design/methodology/approach: To examine the model of the study, design of survey questionnaire was employed through data collected from Dubai Police Departments. Out of 565 questionnaires, 320 only usable returned. Partial Least Square (PLS) structural equation modelling was employed to analyze the data.

Findings: Based on statistical results, the effect of ERP on TQM, organizational excellence, and organizational performance were confirmed. In addition, the effect of TQM and organizational excellence on organizational performance was also confirmed. Moreover, TQM was found to partially mediate the effect of ERP on organizational performance, whereas organizational excellence was found to fully mediate the effect on the same relationship.

Practical implications: The results of this study have several practical implications. This study will help managers and decision makers to take the proper decision when implementing ERP system. Due to that, TQM and organizational excellence are the most important practices to ease the ERP implementation.
\end{abstract}

Originality/value: This study is considered as the only empirical study that examines the collective effect of ERP, TQM, and organizational excellence on organizational performance.

Keywords: enterprise resource planning (ERP), total quality management (TQM), organizational excellence (OE), organizational performance (OP), Dubai Police (DP)

\section{Introduction}

The current global competitive environment compels organizations around the world to enhance and increase their performance through implementing innovative strategies in order to sustain their business and remain competitive. To assist the process of development, organizations have to adopted different philosophies and approaches including Total Quality Management, business excellence (Oakland, 1999), and Enterprise Resource Planning (ERP) (Uwizeyemungu \& Raymond, 2010).

The resource-based view theory (RBV) of the firm has been used in IT business research to investigate and theorize the effect of rare IT capabilities on sustainable competitive advantages (Masli, Richardson, Sanchez, \& Smith, 2010). ERP effects on operations such as in supply chain based on RBV and contingency theories (Hwang \& Min, 2013). Previous research has empirically reported a positive relationship between superior IT capabilities and organizational performance. One of those superior IT systems in the last few years is ERP system. The implementation of ERP as an innovative system with large business organizations, and later expanded to cover other organizations such as small and medium-sized organizations (SMEs) (Everdingen, 
Hillegersberg, \& Waarts, 2000) and organizations of public sector (Kumar, Maheshwari, \& Kumar, 2002). While some organizations reported potential benefits from ERP implementation, others reported horror stories about ERP system (Barker \& Frolick, 2003; Shang \& Seddon, 2000). Therefore, the decision of using the most appropriate system is not an easy task. Organizations have to implement a system that can help them to achieve their goals with low risks. ERP as a complicated system can lead to achieve high performance and competitive advantages if supported by other factors inside and outside the organizations.

Total Quality Management (TQM) as a management philosophy can be one of those factors that can help ERP system to achieve the desired goals. It has been argued that TQM is a pre-requisite practice before implementing ERP system (Ghadilolaee, Aghajani, \& Rahmati, 2010). Excellence as a desired outcome for any strategy and practice can be also considered as a practice that can help organizations to implement the proposed practice in excellent ways to achieve ultimately the best organizational performance. As a result, organizations that demonstrate IT excellence should generate the greatest values from spending in their IT strategy (Masli et al., 2010). In addition, while most of practices in TQM are moving in way, but there are still lacking of the significance of employee role in developing quality improvement for the sake of accomplishing business excellence (Rashid \& Aslam, 2012).

This study examined the relationship between ERP and organizational performance. Due to the inconclusiveness findings in the previous literature of the relationship between ERP and organizational performance, this study is an attempt to investigate the mechanism of TQM and organizational excellence as mediator variables that can explain that relationship through implementing quantitative research based on questionnaire survey.

\section{Related Literature and Research Hypotheses}

The literature review is presented in seven sections. As a base for understanding the other following relationships, the first section presents the relationship between ERP and organizational performance. The other followed sections discussed the relationships between variables in the proposed framework. As a result of the thorough discussion of the previous studies in the literature, several hypotheses are proposed for these relationships to be tested in the following parts of this research.

\subsection{ERP and Organizational Performance}

In the literature there are many definitions for ERP system, however, there is no agreement among researchers on an agreed definition. One of the important definition in the previous literature was defined by Davenport (2002) "ERP as an advanced technological solution system that integrate critical information within organization such as supply chain, finance and accounting, human resource, and customer relationships". In addition, ERP is an information system that integrates the data of the organizations that are used in their operation (Pacheco-Comer \& González-Castolo, 2012). There are many reasons and motivations behind implementation of ERP systems such as technical and business driven implementations (Botta-Genoulaz \& Millet, 2006; Velcu, 2007).

The relationship between ERP system and organizational performance has been examined by many researchers. There are conflicting results in their relationship (Kang, Park, \& Yang, 2008). Some of them reported a positive and significant relationship between ERP and organizational performance (Bendoly \& Kaefer, 2004; da Silveira, Snider, \& Balakrishnan, 2013; Fang \& Lin, 2006; HassabElnaby, Hwang, \& Vonderembse, 2012; Hayes et al., 2001; Hwang \& Min, 2013; Peffers \& Dos Santos, 1996; Velcu, 2007). However, on the other hand, there are some other researchers who reported adverse results (Hitt \& Brynjolfsson, 1996; Weill, 1992; Wier, Hunton, \& HassabElnaby, 2007).

The mixed results in the previous literature of the effect of ERP on organizational performance encourage us to do this study and examine that relationship with the existing of other variables that may help in increasing the positive and significant effect. But before investigating the mediating effect of TQM and organizational excellence, the direct effect of ERP on organizational performance can be examined to compare with other results. Therefore, the following hypothesis is proposed:

\section{$H_{1}$ : Enterprise Resource Planning has a positive and significant effect on the organizational performance.}

\subsection{ERP and TQM}

Implementation of innovative initiatives such as ERP and TQM are considered the most important practices to enhance performance and gain competitive advantages (Abdinnour \& Groen, 2009). There are few studies, such as Laframboise \& Reyes (2005) that discuss the collective effective of both TQM and ERP (Abdinnour \& Groen, 2009). Their study used a qualitative method through interviews in the aerospace industry. In addition, Ghadilolaee, Aghajani, and Rahmati (2010) argued in their study that implementation of ERP should be 
preceded by implementation of TQM practice for the reason that TQM could bring continuous improvement and problem solving that enable organizations to implement ERP successfully.

Most of critical success factors of TQM and ERP are identical such as business process reengineering, culture, learning, training, top management support, open communication, etc. In their contribution to the same field, Jha and Joshi (2007) reported in their study that ERP and TQM practices are considered significant resources for any organization to gain competitive advantage. Additionally, Marc and Gyu (2003) argued that implementation of ERP not always success because of some critical success factors such as TQM, culture, and business process reengineering. From the above discussion, the following hypothesis is postulated:

\section{$H_{2}$ : Enterprise Resource Planning has a positive and significant effect on Total Quality Management}

\subsection{ERP and Organizational Excellence}

The main purpose of implementing ERP in organizations is to achieve the maximum performance and gain competitive advantages over competitors. In order to achieve that, the ERP system should be implemented in excellent ways either through internal or external processes. Organizational excellence is the practice of making organizations better in excellence path and growth (Attafar, Forouzan, \& Shojaei, 2012). According to Moghadami (2005), excellent organizations have different characteristics in terms of customer, employees, leadership, capital owner, learning, future generation, globalization, change or transformation, and suppliers. Each one of these characteristics plays an important role in achieving excellence that leads to achieve the desired performance. When implementing ERP as a technological system, implementers or business owners should focus on the previous characteristics to help ERP to achieve what is planned to be at the earlier stages. To discover the effect of ERP on organizational excellence, the following hypothesis is proposed to be examined:

\section{$H_{3}$ : Enterprise Resource Planning has a positive and significant effect on the organizational Excellence}

\subsection{TQM and Organizational Performance}

Total Quality Management (TQM) is a management philosophy that has been obtained attention by many research (Ehigie \& McAndrew, 2005), and confirms the role of internal and external suppliers, customer, and employees in pursuing continuous improvement (Kanji, 2002). There are a bulk in the literature that show the significance of TQM strategy in manufacturing organizations (Arawati, 2005; Sohal \& Terziovski, 2000), service organizations (Yasin, Kunt, \& Zimmerer, 2004), SMEs organizations (Sohail \& Hoong, 2003), and public organizations (Nor Hazilah, 2004). However, there are plenty of studies on TQM practices, most of them in developed countries and only there are six studies out of 347 were conducted in the Middle East including Saudi Arabia, Qater, and UAE (Sila \& Ebrahimpour, 2002). Therefore, this study is an attempt to fill this gap in the literature.

The relationship between TQM and organizational performance has been examined by many researchers, however, some of them found that TQM can affect and enhance the performance positively and significantly (Abebe, 2014; Barh, Tee, \& Rao , 2002; Dada \& Watson, 2013; Demirbag et al., 2006; Feng, Prajogo, Tan, \& Sohal, 2006; Fotopoulos \& Psomas, 2010; Gadenne \& Sharma, 2009; Hendricks \& Singhal, 1997; Lee , 2004; Salaheldin, 2009; Tang \& Tang, 2012; Wang \& Yen, 2012; Zhang \& Zhang, 2012). On the other hand, other researchers found that TQM has no effect on organizational performance and sometimes may affect the performance negatively (Dooyoung, Kalinowski, \& El-Enein, 1998). The inconsistent findings in the previous literature urge for more researches to be conducted in this area. Therefore this study is a response to those calls from previous researchers to do more researches through including other variables. For this purpose, the following hypothesis is proposed to be tested:

\section{$H_{4}$ : Total Quality Management has a positive and significant effect on the organizational performance.}

\subsection{TQM and Organizational Excellence}

As has been mentioned earlier, TQM is considered as one of the important strategic instrument that helps organizations to achieve optimal performance. In addition, TQM core essentials are to encourage business practice to increase customers' satisfaction, productivity, reduce cost, and enhance quality output. In other words, TQM is a strategy that helps organizations to enhance business excellence (Lee, 2002). According to Ionica and Baleanu (2010), the history of TQM from inspection to business excellence has gone through different stages such as quality control.

There are some studies that investigated the relationship between TQM and organizational excellence such as Sharma and Kodali (2008) who argued that TQM is considered as a fundamental standard to achieve excellence in manufacturing industry. In addition, Ioncia and Baleanu (2010) reported that the underlying principles of 
EFQM Excellence Model are connected with basic TQM's principles. Moreover, Lyons, Acsente, and Waesberghe (2008) examined the relationship between TQM and knowledge management to integrate a sustainable excellence framework. Lee (2002) investigated how business excellence can be sustained through TQM. In conclusion, the area of how TQM can enhance organization performance through excellence is still rare in the literature; therefore this study tries to investigate more about this relationship. To achieve this purpose, the following hypothesis is proposed:

\section{$H_{5}$ : Total Quality Management has a positive and significant effect on the organizational Excellence.}

\subsection{Organizational Excellence and Organizational Performance}

Antony and Bhattacharyya (2010) examined the relationship between organizational excellence and organizational performance where they found that organizational excellence could be figured base on relationship of performance indicators. They found also that organizational excellence enables managers to evaluate their organizations better than organizational performance method. In addition, Ooncharoen and Ussahawanitchakit (2008) in their study found that organizational excellence has a positive and significant effect on performance. Therefore the following hypothesis is postulated to be tested:

\section{$H_{6}$ : Organizational Excellence has a positive and significant effect on the organizational performance.}

\subsection{The Mediating Role of TQM and Organizational Excellence}

As has been stated earlier, the direct effect of ERP on organizational performance has been studied by many researchers. However the abundant studies on this relationship, there are still inconclusiveness about the effect of ERP on organizational performance. Therefore, many researchers call for more researches in this area to be conducted by involving other variables that may affect the relationship positively and significantly. For this purpose, TQM and organizational excellence have been intervened in the relationship as mechanisms to explain in a better way the effect of ERP on organizational performance. Through implementing TQM practices and organizational excellence concepts, ERP may has the power to achieve the desired objectives and enhance performance. TQM and organizational excellence have many dimensions such as leadership, continuous improvement, innovation, customer focus, strategic planning, benchmarking, service design, etc. By practicing these strategies and practices inside the organization, ERP as a new system can benefit in all implementation stages to gain the proposed organizational performance. Logically, TQM and organizational excellence can bring the advantage to organizations because of their involving the elements of success. According to Mele and Colurcio (2006) organizational excellence when linked with TQM has different aspects such as development of partnership, public responsibility, coherence with objectives, leadership, continuous improvement, innovation, and learning. TQM and excellence can complement each other (Adebanjo, 2001), and therefore can bring a powerful management tools to facilitate the implementation of ERP system for the sake of achieving high organizational performance. In addition, the mediation of TQM as a strategic resource was examined by Prajogo and Sohal (2006) between differentiation strategy and performance where they found a partial mediation.

For this purpose, the following hypotheses are preoposed to be examined in the next stage:

\section{$H_{7}:$ TQM mediate the relationship between ERP and Organizational Performance}

\section{$H_{8}$ : Organizational Excellence mediate the relationship between ERP and Organizational Performance}

\section{Methodology}

The main purpose of this study is to examine the effect of variables on each other's. To achieve that a quantitative methodology approach was employed. Questionnaire survey is considered as one of the important instruments to collect the primary data from respondents (Kerlinger \& Lee, 2000). The data collected through cross-sectional research design which is suitable for this study to collect the data at the point of time. The survey questionnaires were distributed in January, 2014 and collected end of February, 2014 through hard copy and emails. The source of data was Dubai Police where the first author works. Because of the nature of this study's variables, sections of Dubai Police have been selected to fill the questionnaire through Head section officers. Head section officers are the middle managers as a link between managers and employees. They know better than other on how these practices and strategies are working. Five hundred forty five questionnaires have been distributed and three hundred and twenty were returned completely. Dimensions and measurements have been adopted and adapted from previous studies. ERP measurements have been adopted from Stratman and Roth (2002), whereas measurements of TQM from different sources: Brah, Wong, and Rao (2000), Anderson and Sohal (1999), Terziovski \& Samson (1999), and Rao (2000). Measurements of organizational excellence have been adopted from Pinar and Girard (2000), and organizational performance from Kaplan and Norton (1992; 2000). Structural Equation Modeling (SEM) is the technique that used to analyze the data and test the proposed 
hypothesis through Smart-PLS statistical software.

\section{Statistical Analysis and Results}

Partial Least Square (PLS) is used to confirm the reliability and validity of the outer. As confirmed by many researchers, reliability and validity are the initial tests before doing the hypotheses testing. The model of this study contains the variables: ERP, TQM, organizational excellence and organizational performance. In order to examine the relationships between these variables, this study follows the two-step approach suggested by Chin (1998).

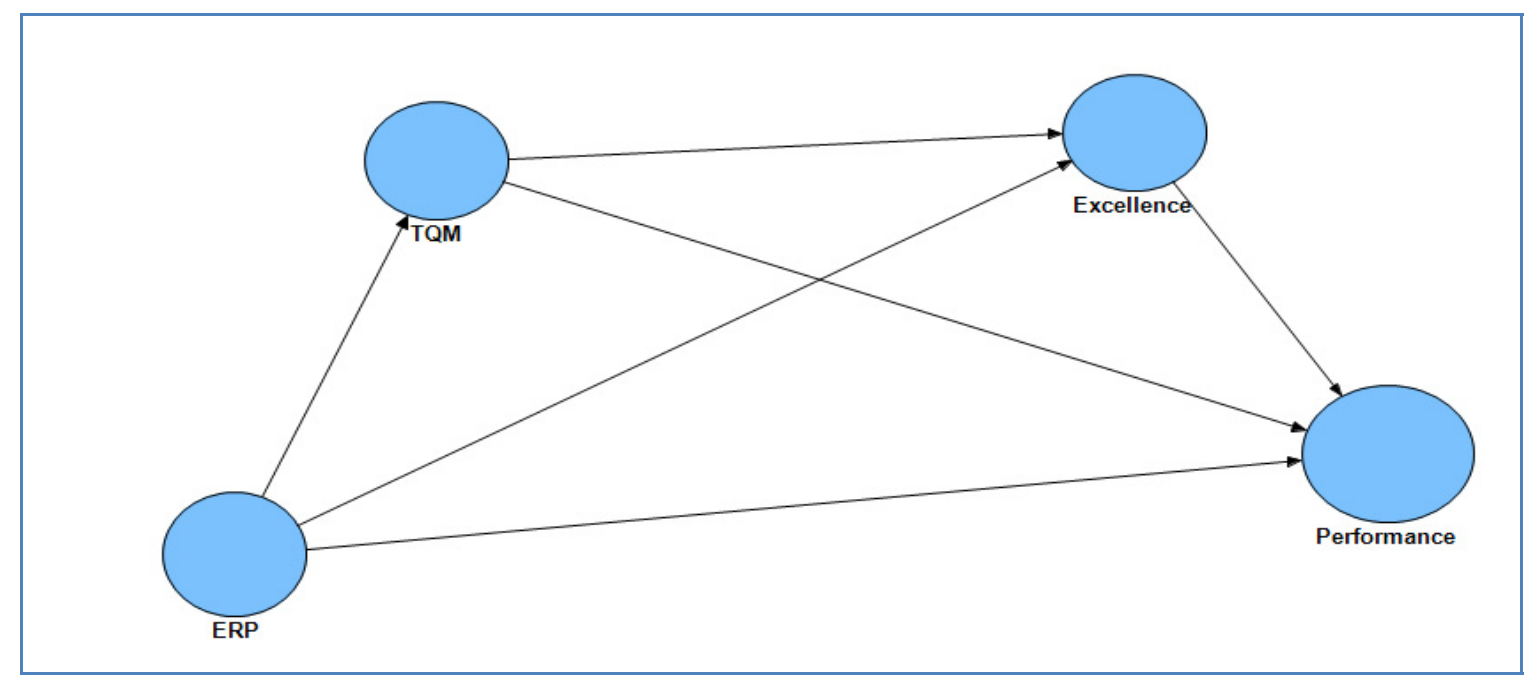

Figure 1. The research framework

In the literature of Structural Equation Modeling (SEM) the construct validity and reliability of the model should be approved before examining the hypothesized relationships.

\subsection{The Outer Model (Measurement)}

The following sections test the validity and reliability of the constructs before establishing the goodness of the measurement model. The construct validity and reliability was tested through the content validity, the discriminant validity, and the convergent validity as illustrated in the next sections.

\subsubsection{The Content Validity}

In multivariate analysis literature, the content validity of the construct compared with the other model's constructs. Therefore, Chin (1998) and Hair et al. (2010) suggested the using of factor loading to examine the content validity. To do that, the items will be deleted if are loaded high with other constructs than their respective ones. Table 1 and 2 showed that all the constructs are significantly loaded higher in their respective variables. Therefore, these results confirmed that the measurement model of this study has content validity.

Table 1. Factor loading significance

\begin{tabular}{llllll}
\hline Construct & Items & Loadings & Standard Error & T Value & P Value \\
\hline \multirow{3}{*}{ Benchmarking } & B1 & $\mathbf{0 . 9 2 8}$ & $\mathbf{0 . 0 1 5}$ & $\mathbf{6 1 . 9 4 5}$ & 0.000 \\
& B2 & $\mathbf{0 . 8 8 4}$ & $\mathbf{0 . 0 2 1}$ & $\mathbf{4 3 . 0 7 1}$ & 0.000 \\
& B3 & $\mathbf{0 . 8 7 7}$ & $\mathbf{0 . 0 2 5}$ & $\mathbf{3 4 . 7 9 5}$ & 0.000 \\
Continuous Improvement & CI1 & $\mathbf{0 . 9 0 2}$ & $\mathbf{0 . 0 1 2}$ & $\mathbf{7 6 . 1 1 7}$ & 0.000 \\
& CI2 & $\mathbf{0 . 9 2 6}$ & $\mathbf{0 . 0 0 7}$ & $\mathbf{1 2 7 . 9 1 2}$ & 0.000 \\
& CI3 & $\mathbf{0 . 8 6 7}$ & $\mathbf{0 . 0 2 0}$ & $\mathbf{4 3 . 0 5 8}$ & 0.000 \\
ERP-Business Process Skills & CI4 & $\mathbf{0 . 8 7 5}$ & $\mathbf{0 . 0 1 6}$ & $\mathbf{5 5 . 4 2 4}$ & 0.000 \\
& ERPB1 & $\mathbf{0 . 8 5 1}$ & $\mathbf{0 . 0 1 8}$ & $\mathbf{4 6 . 6 4 2}$ & 0.000 \\
& ERPB2 & $\mathbf{0 . 8 7 6}$ & $\mathbf{0 . 0 1 2}$ & $\mathbf{7 1 . 1 4 3}$ & 0.000 \\
\hline
\end{tabular}




\begin{tabular}{|c|c|c|c|c|c|}
\hline Construct & Items & Loadings & Standard Error & T Value & P Value \\
\hline \multirow{8}{*}{ ERP-Change Readiness } & ERPB4 & 0.919 & 0.011 & 84.528 & 0.000 \\
\hline & ERPB5 & 0.763 & 0.030 & 25.113 & 0.000 \\
\hline & ERPC1 & 0.861 & 0.018 & 46.858 & 0.000 \\
\hline & ERPC2 & 0.932 & 0.014 & 65.883 & 0.000 \\
\hline & ERPC3 & 0.880 & 0.012 & 72.873 & 0.000 \\
\hline & ERPC4 & 0.942 & 0.006 & 150.155 & 0.000 \\
\hline & ERPC5 & 0.888 & 0.013 & 70.955 & 0.000 \\
\hline & ERPE1 & 0.861 & 0.023 & 37.969 & 0.000 \\
\hline \multirow{4}{*}{ ERP-Executive Commitment } & ERPE2 & 0.909 & 0.012 & 77.958 & 0.000 \\
\hline & ERPE3 & 0.925 & 0.009 & 99.169 & 0.000 \\
\hline & ERPE4 & 0.911 & 0.013 & 70.299 & 0.000 \\
\hline & ERPE5 & 0.714 & 0.033 & 21.507 & 0.000 \\
\hline \multirow{5}{*}{ ERP-IT Skills } & ERPI1 & 0.840 & 0.023 & 35.784 & 0.000 \\
\hline & ERPI2 & 0.880 & 0.015 & 60.673 & 0.000 \\
\hline & ERPI3 & 0.891 & 0.015 & 59.710 & 0.000 \\
\hline & ERPI4 & 0.934 & 0.008 & 114.491 & 0.000 \\
\hline & ERPI5 & 0.817 & 0.031 & 26.168 & 0.000 \\
\hline \multirow{5}{*}{ ERP-Learning } & ERPL1 & 0.778 & 0.024 & 31.882 & 0.000 \\
\hline & ERPL2 & 0.811 & 0.027 & 30.497 & 0.000 \\
\hline & ERPL3 & 0.851 & 0.015 & 57.700 & 0.000 \\
\hline & ERPL4 & 0.894 & 0.010 & 85.273 & 0.000 \\
\hline & ERPL5 & 0.842 & 0.022 & 38.850 & 0.000 \\
\hline \multirow{5}{*}{ ERP-Project Management } & ERPP1 & 0.903 & 0.013 & 69.904 & 0.000 \\
\hline & ERPP2 & 0.905 & 0.012 & 76.008 & 0.000 \\
\hline & ERPP3 & 0.895 & 0.014 & 64.196 & 0.000 \\
\hline & ERPP4 & 0.895 & 0.013 & 67.671 & 0.000 \\
\hline & ERPP5 & 0.755 & 0.032 & 23.421 & 0.000 \\
\hline \multirow{5}{*}{ ERP-Strategic IT Planning } & ERPS1 & 0.840 & 0.015 & 55.988 & 0.000 \\
\hline & ERPS2 & 0.887 & 0.012 & 77.035 & 0.000 \\
\hline & ERPS3 & 0.901 & 0.012 & 72.709 & 0.000 \\
\hline & ERPS4 & 0.904 & 0.013 & 69.108 & 0.000 \\
\hline & ERPS5 & 0.862 & 0.018 & 46.898 & 0.000 \\
\hline \multirow{5}{*}{ ERP-Training } & ERPT1 & 0.825 & 0.020 & 41.460 & 0.000 \\
\hline & ERPT2 & 0.766 & 0.032 & 24.074 & 0.000 \\
\hline & ERPT3 & 0.850 & 0.016 & 54.514 & 0.000 \\
\hline & ERPT4 & 0.847 & 0.018 & 47.484 & 0.000 \\
\hline & ERPT5 & 0.805 & 0.021 & 37.848 & 0.000 \\
\hline \multirow{3}{*}{ Excellence-Customer Focus } & EXC1 & 0.927 & 0.011 & 84.758 & 0.000 \\
\hline & EXC2 & 0.951 & 0.008 & 119.483 & 0.000 \\
\hline & EXC3 & 0.933 & 0.009 & 99.535 & 0.000 \\
\hline \multirow{3}{*}{ Excellence-Innovation } & EXI1 & 0.884 & 0.014 & 61.997 & 0.000 \\
\hline & EXI2 & 0.908 & 0.015 & 62.436 & 0.000 \\
\hline & EXI3 & 0.910 & 0.013 & 68.133 & 0.000 \\
\hline \multirow{4}{*}{ Excellence-Personnel Commitment } & EXP1 & 0.855 & 0.017 & 50.163 & 0.000 \\
\hline & EXP2 & 0.862 & 0.020 & 43.673 & 0.000 \\
\hline & EXP3 & 0.837 & 0.025 & 33.668 & 0.000 \\
\hline & EXP4 & 0.802 & 0.028 & 28.481 & 0.000 \\
\hline \multirow{4}{*}{ HRM } & HRE1 & 0.862 & 0.015 & 57.933 & 0.000 \\
\hline & HRE2 & 0.785 & 0.020 & 38.587 & 0.000 \\
\hline & HRE3 & 0.785 & 0.020 & 39.039 & 0.000 \\
\hline & HRI1 & 0.793 & 0.028 & 28.688 & 0.000 \\
\hline
\end{tabular}




\begin{tabular}{|c|c|c|c|c|c|}
\hline Construct & Items & Loadings & Standard Error & T Value & P Value \\
\hline \multirow[b]{8}{*}{ Information and Analysis } & HRI2 & 0.844 & 0.020 & 42.946 & 0.000 \\
\hline & HRI3 & 0.889 & 0.013 & 69.151 & 0.000 \\
\hline & HRT2 & 0.776 & 0.026 & 30.201 & 0.000 \\
\hline & HRT3 & 0.905 & 0.011 & 82.948 & 0.000 \\
\hline & HRTI & 0.860 & 0.014 & 61.412 & 0.000 \\
\hline & IA1 & 0.801 & 0.024 & 32.802 & 0.000 \\
\hline & IA2 & 0.910 & 0.011 & 82.811 & 0.000 \\
\hline & IA3 & 0.848 & 0.022 & 38.033 & 0.000 \\
\hline \multirow{6}{*}{ Management Leadership } & IA4 & 0.888 & 0.014 & 64.433 & 0.000 \\
\hline & IA5 & 0.902 & 0.010 & 86.148 & 0.000 \\
\hline & ML1 & 0.924 & 0.010 & 93.907 & 0.000 \\
\hline & ML2 & 0.892 & 0.017 & 51.406 & 0.000 \\
\hline & ML3 & 0.938 & 0.009 & 104.723 & 0.000 \\
\hline & ML4 & 0.920 & 0.012 & 78.487 & 0.000 \\
\hline \multirow{4}{*}{ Customer } & OPC4 & 0.799 & 0.010 & 85.559 & 0.000 \\
\hline & OPC5 & 0.824 & 0.020 & 40.056 & 0.000 \\
\hline & OPC6 & 0.830 & 0.033 & 21.607 & 0.000 \\
\hline & OPC7 & 0.777 & 0.027 & 28.825 & 0.000 \\
\hline \multirow{3}{*}{ Financial } & OPF1 & 0.866 & 0.020 & 40.355 & 0.000 \\
\hline & OPF2 & 0.740 & 0.027 & 28.253 & 0.000 \\
\hline & OPF3 & 0.762 & 0.013 & 64.619 & 0.000 \\
\hline \multirow{4}{*}{ Internal Process } & OPI10 & 0.794 & 0.042 & 17.748 & 0.000 \\
\hline & OPI11 & 0.719 & 0.044 & 17.473 & 0.000 \\
\hline & OPI8 & 0.822 & 0.033 & 24.584 & 0.000 \\
\hline & OPI9 & 0.823 & 0.023 & 35.240 & 0.000 \\
\hline \multirow{4}{*}{ Learning and Growth } & OPL12 & 0.771 & 0.024 & 34.033 & 0.000 \\
\hline & OPL13 & 0.803 & 0.022 & 35.931 & 0.000 \\
\hline & OPL14 & 0.754 & 0.019 & 44.190 & 0.000 \\
\hline & OPL15 & 0.869 & 0.020 & 41.264 & 0.000 \\
\hline \multirow{3}{*}{ Service Design } & SD1 & 0.892 & 0.014 & 63.562 & 0.000 \\
\hline & SD2 & 0.943 & 0.007 & 140.834 & 0.000 \\
\hline & SD3 & 0.890 & 0.009 & 96.015 & 0.000 \\
\hline \multirow{4}{*}{ Stratgic Planning } & SP1 & 0.841 & 0.018 & 46.320 & 0.000 \\
\hline & SP2 & 0.884 & 0.014 & 63.567 & 0.000 \\
\hline & SP3 & 0.894 & 0.011 & 81.630 & 0.000 \\
\hline & SP4 & 0.846 & 0.015 & 56.679 & 0.000 \\
\hline
\end{tabular}

Table 2. Factor analysis results

\begin{tabular}{|c|c|c|c|c|c|c|c|c|c|c|c|c|c|c|c|c|c|c|c|c|c|c|c|c|}
\hline $\begin{array}{l}\text { Constr } \\
\text { uct }\end{array}$ & Е્气 & 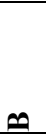 & 0 & 点 & $\begin{array}{l}\cup \\
\underline{0} \\
\underline{01}\end{array}$ & $\frac{\underline{a}}{0}$ & $\underline{a}$ & $\underline{\underline{2}}$ & $\frac{\hat{a}}{\hat{a}}$ & $\begin{array}{l}\tilde{A} \\
\hat{a} \\
\underline{a}\end{array}$ & 占 & 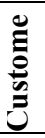 & 율 & $=0$ & ఏ & $\underline{\underline{\underline{x}}}$ & $\lesssim$ & $\sum$ & อ & $\frac{1}{0}$ & $\overline{0}$ & $\frac{1}{0}$ & $\hat{\mathscr{L}}$ & $\hat{\sigma}$ \\
\hline \multirow{6}{*}{$\begin{array}{l}\text { Benchm } \\
\text { arking }\end{array}$} & \multirow{2}{*}{ B1 } & 0. & 0. & 0. & 0. & 0. & 0. & 0. & 0. & 0. & 0. & 0. & & 0. & 0. & 0. & 0. & 0. & 0. & 0. & 0. & 0. & 0. & 0. \\
\hline & & 9 & 8 & 55 & 37 & 72 & 53 & 5 & 45 & 73 & 5 & 45 & & 49 & 28 & 75 & 71 & 47 & 35 & 16 & 32 & 35 & 72 & 61 \\
\hline & \multirow{2}{*}{ B2 } & 0. & 0. & 0. & 0. & 0. & 0. & 0. & 0. & 0. & 0. & 0 . & & 0. & 0. & 0. & 0. & 0. & 0. & 0. & 0. & 0. & 0. & \#\# \\
\hline & & 9 & 74 & 56 & 36 & 57 & 56 & 45 & 42 & 7 & 47 & 32 & & 47 & 3 & 76 & 73 & 46 & 26 & 17 & 29 & 35 & 71 & \# \\
\hline & \multirow{2}{*}{ B3 } & 0. & 0. & 0. & 0. & 0. & 0. & 0. & 0. & 0. & 0. & 0 . & & 0. & 0. & 0. & 0. & 0. & 0. & 0. & 0. & 0. & 0. & 0. \\
\hline & & 9 & 73 & 52 & 27 & 59 & 52 & 4 & 4 & 71 & 41 & 36 & & 51 & 36 & 67 & 71 & 52 & 25 & 16 & 3 & 38 & 68 & 58 \\
\hline \multirow{4}{*}{$\begin{array}{l}\text { Continu } \\
\text { ous } \\
\text { Improv } \\
\text { ement }\end{array}$} & \multirow{2}{*}{ CI1 } & 0. & 0. & 0. & 0. & 0. & 0. & 0. & 0. & 0. & 0. & 0 . & & 0. & 0. & 0. & 0. & 0. & 0. & 0. & 0. & 0. & 0. & 0. \\
\hline & & 76 & 9 & 62 & 57 & 75 & 63 & 61 & 59 & 75 & 6 & 53 & & 6 & 5 & 77 & 86 & 55 & 41 & 39 & 48 & 52 & 82 & 66 \\
\hline & CI2 & 0. & 0. & 0. & 0. & 0. & 0. & 0. & 0. & 0. & 0. & 0. & & 0. & 0. & 0. & 0. & 0. & 0. & 0. & 0. & 0. & 0. & 0. \\
\hline & 212 & 77 & 9 & 61 & 47 & 74 & 58 & 52 & 61 & 78 & 49 & 41 & & 61 & 49 & 78 & 81 & 67 & 33 & 37 & 45 & 49 & 8 & 69 \\
\hline
\end{tabular}




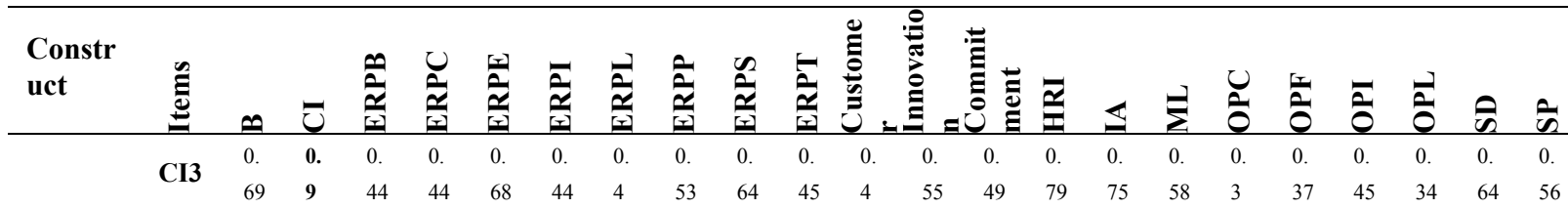

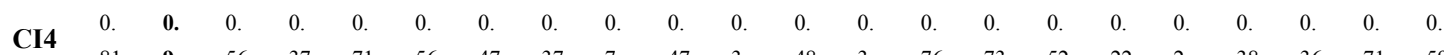

$\begin{array}{lllllllllllllllllllllllll}\text { ER } & 0 . & 0 . & 0 . & 0 . & 0 . & 0 . & 0 . & 0 . & 0 . & 0 . & 0 . & 0 . & 0 . & 0 . & 0 . & 0 . & 0 . & 0 . & 0 . & 0 . & 0 . & 0 .\end{array}$

$\begin{array}{llllllllllllllllllllllllll}\text { PB1 } & 44 & 48 & 9 & 66 & 52 & 86 & 76 & 5 & 58 & 73 & 25 & 45 & 26 & 49 & 5 & 3 & 25 & 12 & 29 & 38 & 52 & 48\end{array}$

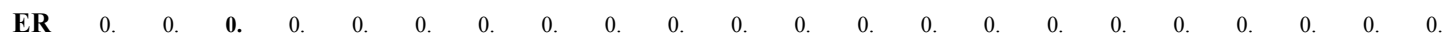

$\begin{array}{lllllllllllllllllllllllllll}\text { PB2 } & 6 & 57 & 9 & 6 & 64 & 84 & 74 & 53 & 64 & 68 & 42 & 49 & 25 & 55 & 5 & 3 & 39 & 14 & 31 & 43 & 57 & 54\end{array}$

ERPB $\quad \begin{array}{llllllllllllllllllllllll} & \text { ER } & 0 . & 0 . & 0 . & 0 . & 0 . & 0 . & 0 . & 0 . & 0 . & 0 . & 0 . & 0 . & 0 . & 0 . & 0 . & 0 . & 0 . & 0 . & 0 . & 0 . & 0 . & 0 .\end{array}$

$\begin{array}{lllllllllllllllllllllllllll}\text { PB3 } & 51 & 5 & 9 & 56 & 55 & 71 & 64 & 49 & 54 & 61 & 51 & 47 & 36 & 56 & 52 & 25 & 51 & 07 & 27 & 43 & 48 & 39\end{array}$

$\begin{array}{llllllllllllllllllllllll}\text { ER } & 0 . & 0 . & \mathbf{0 .} & 0 . & 0 . & 0 . & 0 . & 0 . & 0 . & 0 . & 0 . & 0 . & 0 . & 0 . & 0 . & 0 . & 0 . & 0 . & 0 . & 0 . & 0 . & 0 . \\ \text { PB4 } & 52 & 58 & \mathbf{9} & 64 & 57 & 76 & 71 & 58 & 65 & 68 & 41 & 52 & 34 & 63 & 59 & 31 & 38 & 2 & 34 & 42 & 59 & 44\end{array}$

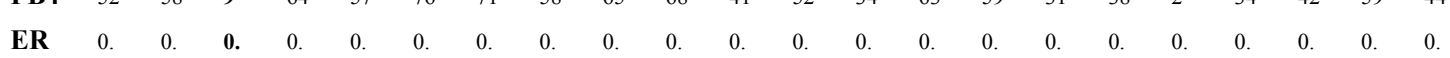

$\begin{array}{lllllllllllllllllllllll}\text { PB5 } & 49 & 57 & \mathbf{8} & 61 & 52 & 59 & 58 & 45 & 55 & 67 & 31 & 49 & 32 & 6 & 56 & 33 & 25 & 25 & 31 & 35 & 49 & 35\end{array}$

$\begin{array}{lllllllllllllllllllllll}\text { ER } & 0 . & 0 . & 0 . & \mathbf{0 .} & 0 . & 0 . & 0 . & 0 . & 0 . & 0 . & 0 . & 0 . & 0 . & 0 . & 0 . & 0 . & 0 . & 0 . & 0 . & 0 . & 0 . & 0 .\end{array}$

$\begin{array}{lllllllllllllllllllllll}\text { PC } & 0 . & 0 . & 0 . & \mathbf{0 .} & 0 . & 0 . & 0 . & 0 . & 0 . & 0 . & 0 . & 0 . & 0 . & 0 . & 0 . & 0 . & 0 . & 0 . & 0 . & 0 . & 0 . & 0 . \\ \mathbf{1} & 41 & 52 & 68 & \mathbf{9} & 54 & 73 & 79 & 67 & 43 & 67 & 39 & 43 & 48 & 43 & 55 & 5 & 47 & 4 & 24 & 46 & 5 & 56\end{array}$

$\begin{array}{lllllllllllllllllllllllll}\text { ER } & 0 . & 0 . & 0 . & \mathbf{0 .} & 0 . & 0 . & 0 . & 0 . & 0 . & 0 . & 0 . & 0 . & 0 . & 0 . & 0 . & 0 . & 0 . & 0 . & 0 . & 0 . & 0 . & 0 .\end{array}$

$\begin{array}{lllllllllllllllllllllllllll}\mathbf{2} & 26 & 39 & 63 & \mathbf{9} & 4 & 63 & 77 & 61 & 33 & 68 & 38 & 41 & 44 & 37 & 47 & 37 & 38 & 36 & 24 & 33 & 37 & 42\end{array}$

$\begin{array}{llllllllllllllllllllllll}\text { ER } & 0 . & 0 . & 0 . & 0 . & 0 . & 0 . & 0 . & 0 . & 0 . & 0 . & 0 . & 0 . & 0 . & 0 . & 0 . & 0 . & 0 . & 0 . & 0 . & 0 . & 0 . & 0 .\end{array}$

$\begin{array}{llllllllllllllllllllllllll}\text { ERPC } & \text { PC } & 0 . & 0 . & 0 . & \mathbf{0 .} & 0 . & 0 . & 0 . & 0 . & 0 . & 0 . & 0 . & 0 . & 0 . & 0 . & 0 . & 0 . & 0 . & 0 . & 0 . & 0 . & 0 . & 0 . \\ & 3 & 34 & 45 & 57 & \mathbf{9} & 47 & 61 & 75 & 58 & 39 & 68 & 34 & 39 & 4 & 4 & 47 & 4 & 24 & 36 & 21 & 25 & 4 & 43\end{array}$

$\begin{array}{llllllllllllllllllllllll}\text { ER } & 0 . & 0 . & 0 . & 0 . & 0 . & 0 . & 0 . & 0 . & 0 . & 0 . & 0 . & 0 . & 0 . & 0 . & 0 . & 0 . & 0 . & 0 . & 0 . & 0 . & 0 . & 0 .\end{array}$

$\begin{array}{lllllllllllllllllllllllll}\mathbf{P C} & 29 & 48 & 68 & \mathbf{9} & 5 & 69 & 81 & 63 & 38 & 72 & 37 & 44 & 46 & 41 & 51 & 41 & 32 & 34 & 24 & 34 & 46 & 42\end{array}$

$\begin{array}{llllllllllllllllllllllllll}\text { ER } & 0 . & 0 . & 0 . & \mathbf{0 .} & 0 . & 0 . & 0 . & 0 . & 0 . & 0 . & 0 . & 0 . & 0 . & 0 . & 0 . & 0 . & 0 . & 0 . & 0 . & 0 . & 0 . & 0 .\end{array}$

$\begin{array}{lllllllllllllllllllllllll}5 & 4 & 49 & 65 & 9 & 57 & 64 & 8 & 61 & 46 & 72 & 33 & 41 & 38 & 44 & 53 & 5 & 32 & 37 & 21 & 36 & 51 & 5\end{array}$

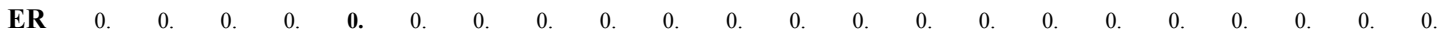

$\begin{array}{lllllllllllllllllllllllll}\text { PE1 } & 57 & 7 & 6 & 54 & 9 & 6 & 52 & 5 & 73 & 56 & 35 & 48 & 35 & 63 & 58 & 63 & 29 & 38 & 36 & 39 & 62 & 67\end{array}$

$\begin{array}{llllllllllllllllllllllll}\text { ER } & 0 . & 0 . & 0 . & 0 . & \mathbf{0 .} & 0 . & 0 . & 0 . & 0 . & 0 . & 0 . & 0 . & 0 . & 0 . & 0 . & 0 . & 0 . & 0 . & 0 . & 0 . & 0 . & 0 .\end{array}$

$\begin{array}{llllllllllllllllllllllllllll}\text { PE2 } & 66 & 77 & 63 & 48 & 9 & 59 & 55 & 48 & 78 & 6 & 41 & 45 & 31 & 7 & 65 & 59 & 37 & 26 & 37 & 44 & 68 & 65\end{array}$

$\begin{array}{llllllllllllllllllllllllll}\text { ERPE } & \text { ER } & 0 . & 0 . & 0 . & 0 . & 0 . & 0 . & 0 . & 0 . & 0 . & 0 . & 0 . & 0 . & 0 . & 0 . & 0 . & 0 . & 0 . & 0 . & 0 . & 0 . & 0 . & 0 .\end{array}$

$\begin{array}{lllllllllllllllllllllllllll}\text { PE3 } & 73 & 74 & 57 & 41 & 9 & 49 & 49 & 53 & 79 & 5 & 48 & 46 & 31 & 7 & 63 & 58 & 48 & 28 & 38 & 48 & 69 & 68\end{array}$

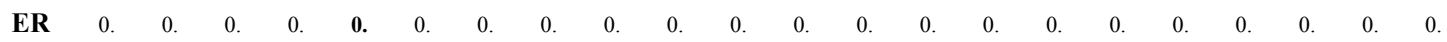

$\begin{array}{lllllllllllllllllllllllllll}\text { PE4 } & 69 & 67 & 5 & 39 & 9 & 45 & 41 & 48 & 73 & 49 & 44 & 5 & 35 & 62 & 57 & 6 & 42 & 34 & 37 & 48 & 65 & 67\end{array}$

$\begin{array}{lllllllllllllllllllllllll}\text { ER } & 0 . & 0 . & 0 . & 0 . & \mathbf{0 .} & 0 . & 0 . & 0 . & 0 . & 0 . & 0 . & 0 . & 0 . & 0 . & 0 . & 0 . & 0 . & 0 . & 0 . & 0 . & 0 . & 0 .\end{array}$

$\begin{array}{llllllllllllllllllllllllllll}\text { PE5 } & 46 & 61 & 52 & 56 & 7 & 51 & 55 & 79 & 61 & 42 & 53 & 47 & 5 & 49 & 58 & 55 & 43 & 46 & 3 & 47 & 68 & 57\end{array}$

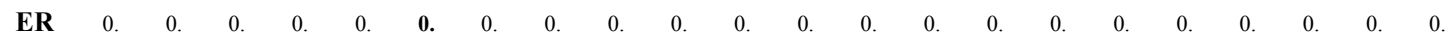

$\begin{array}{lllllllllllllllllllllll}\text { PI1 } & 53 & 62 & 72 & 77 & 61 & \mathbf{8} & 74 & 68 & 6 & 61 & 4 & 48 & 45 & 5 & 63 & 53 & 37 & 38 & 24 & 49 & 65 & 59\end{array}$

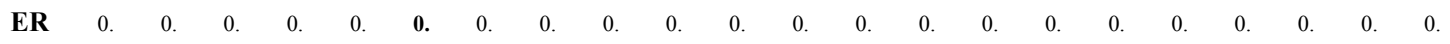

$\begin{array}{llllllllllllllllllllllllll}\text { PI2 } & 49 & 46 & 71 & 57 & 42 & \mathbf{9} & 68 & 45 & 56 & 64 & 21 & 45 & 24 & 48 & 51 & 3 & 23 & 15 & 33 & 41 & 49 & 48\end{array}$

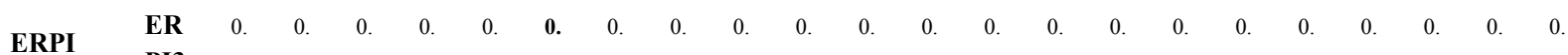

$\begin{array}{llllllllllllllllllllllllllll}\text { PI3 } & 58 & 59 & 75 & 59 & 54 & \mathbf{9} & 67 & 48 & 56 & 7 & 34 & 41 & 28 & 58 & 59 & 31 & 34 & 15 & 35 & 44 & 57 & 53\end{array}$

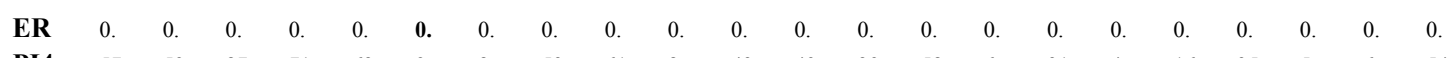

$\begin{array}{llllllllllllllllllllllllll}\text { PI4 } & 57 & 59 & 87 & 71 & 62 & \mathbf{9} & 8 & 53 & 61 & 8 & 43 & 49 & 29 & 58 & 6 & 31 & 4 & 16 & 35 & 5 & 6 & 54\end{array}$

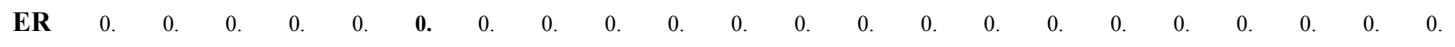

$\begin{array}{lllllllllllllllllllllllll}\text { PI5 } & 39 & 43 & 79 & 57 & 46 & \mathbf{8} & 7 & 49 & 56 & 68 & 29 & 52 & 3 & 44 & 44 & 25 & 25 & 16 & 34 & 39 & 47 & 42\end{array}$

$\begin{array}{llllllllllllllllllllllllll}\text { ER } & 0 . & 0 . & 0 . & 0 . & 0 . & 0 . & \mathbf{0 .} & 0 . & 0 . & 0 . & 0 . & 0 . & 0 . & 0 . & 0 . & 0 . & 0 . & 0 . & 0 . & 0 . & 0 . & 0 .\end{array}$

$\begin{array}{llllllllllllllllllllllllll}\text { PL1 } & 45 & 43 & 69 & 6 & 48 & 73 & \mathbf{8} & 47 & 54 & 74 & 3 & 37 & 23 & 47 & 51 & 27 & 29 & 14 & 24 & 37 & 5 & 49\end{array}$

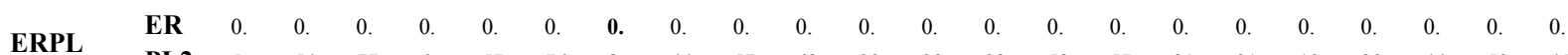

$\begin{array}{lllllllllllllllllllllllllll}\text { PL2 } & 5 & 54 & 77 & 6 & 57 & 76 & \mathbf{8} & 44 & 57 & 68 & 23 & 38 & 23 & 58 & 57 & 31 & 31 & 13 & 29 & 44 & 59 & 45\end{array}$

$\begin{array}{lllllllllllllllllllllll}\text { ER } & 0 . & 0 . & 0 . & 0 . & 0 . & 0 . & \mathbf{0 .} & 0 . & 0 . & 0 . & 0 . & 0 . & 0 . & 0 . & 0 . & 0 . & 0 . & 0 . & 0 . & 0 . & 0 . & 0 .\end{array}$ 


\section{Constr} uct

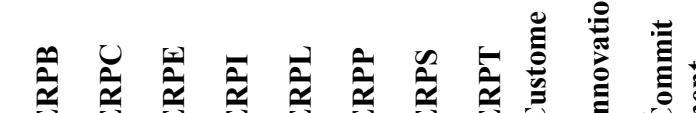

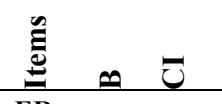

$\begin{array}{lllllll}\text { ER } & 0 . & 0 . & 0 . & 0 . & 0 . & 0 .\end{array}$

$\begin{array}{lllllllll}\text { PL4 } & 39 & 44 & 62 & 8 & 44 & 67 & 9 & 59\end{array}$

$\begin{array}{llllllllllllllllllllllll}\text { ER } & 0 . & 0 . & 0 . & 0 . & 0 . & 0 . & \mathbf{0 .} & 0 . & 0 . & 0 . & 0 . & 0 . & 0 . & 0 . & 0 . & 0 . & 0 . & 0 . & 0 . & 0 . & 0 . & 0 .\end{array}$

$\begin{array}{lllllllllllllllllllllllll}\text { PL5 } & 43 & 47 & 6 & 78 & 45 & 65 & \mathbf{8} & 53 & 39 & 6 & 28 & 29 & 3 & 38 & 53 & 35 & 25 & 2 & 05 & 26 & 49 & 41\end{array}$

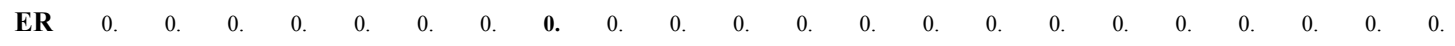

$\begin{array}{lllllllllllllllllllllllllll}\text { PP1 } & 36 & 48 & 55 & 59 & 52 & 59 & 55 & \mathbf{9} & 43 & 37 & 4 & 6 & 65 & 47 & 54 & 59 & 44 & 46 & 43 & 54 & 59 & 56\end{array}$

$\begin{array}{llllllllllllllllllllllll}\text { ER } & 0 . & 0 . & 0 . & 0 . & 0 . & 0 . & 0 . & \mathbf{0 .} & 0 . & 0 . & 0 . & 0 . & 0 . & 0 . & 0 . & 0 . & 0 . & 0 . & 0 . & 0 . & 0 . & 0 .\end{array}$

$\begin{array}{lllllllllllllllllllllllllll}\text { PP2 } & 45 & 55 & 49 & 62 & 56 & 55 & 56 & \mathbf{9} & 45 & 41 & 39 & 55 & 57 & 51 & 58 & 6 & 41 & 49 & 42 & 54 & 63 & 61\end{array}$

$\begin{array}{lllllllllllllllllllllllll}\text { ERPP } & \text { ER } & 0 . & 0 . & 0 . & 0 . & 0 . & 0 . & 0 . & \mathbf{0 .} & 0 . & 0 . & 0 . & 0 . & 0 . & 0 . & 0 . & 0 . & 0 . & 0 . & 0 . & 0 . & 0 . & 0 .\end{array}$

$\begin{array}{llllllllllllllllllllllll}\text { PP3 } & 45 & 51 & 56 & 66 & 64 & 56 & 64 & \mathbf{9} & 52 & 43 & 51 & 48 & 49 & 42 & 5 & 54 & 5 & 48 & 33 & 53 & 6 & 58\end{array}$

$\begin{array}{lllllllllllllllllllllll}\text { ER } & 0 . & 0 . & 0 . & 0 . & 0 . & 0 . & 0 . & \mathbf{0 .} & 0 . & 0 . & 0 . & 0 . & 0 . & 0 . & 0 . & 0 . & 0 . & 0 . & 0 . & 0 . & 0 . & 0 . \\ \text { PP4 } & 46 & 54 & 55 & 61 & 59 & 54 & 54 & \mathbf{9} & 49 & 41 & 49 & 52 & 55 & 55 & 59 & 55 & 53 & 51 & 36 & 52 & 59 & 54\end{array}$

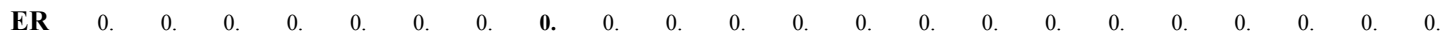

$\begin{array}{llllllllllllllllllllllllllll}\text { PP5 } & 35 & 51 & 44 & 53 & 47 & 4 & 41 & \mathbf{8} & 43 & 42 & 45 & 58 & 64 & 49 & 56 & 5 & 4 & 56 & 35 & 45 & 48 & 37\end{array}$

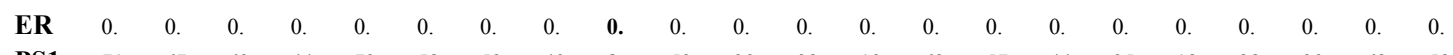

$\begin{array}{lllllllllllllllllllllllllll}\text { PS1 } & 71 & 67 & 63 & 44 & 73 & 58 & 52 & 49 & \mathbf{8} & 53 & 39 & 38 & 19 & 63 & 57 & 44 & 35 & 18 & 28 & 39 & 62 & 58\end{array}$

$\begin{array}{lllllllllllllllllllllllll}\text { ER } & 0 . & 0 . & 0 . & 0 . & 0 . & 0 . & 0 . & 0 . & \mathbf{0 .} & 0 . & 0 . & 0 . & 0 . & 0 . & 0 . & 0 . & 0 . & 0 . & 0 . & 0 . & 0 . & 0 .\end{array}$

$\begin{array}{lllllllllllllllllllllllllll}\text { PS2 } & 7 & 71 & 65 & 37 & 76 & 63 & 51 & 52 & 9 & 52 & 45 & 52 & 34 & 7 & 66 & 48 & 38 & 19 & 34 & 46 & 72 & 59\end{array}$

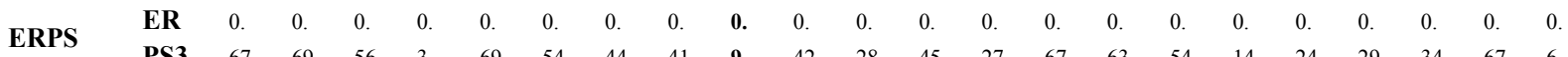

$\begin{array}{llllllllllllllllllllllllll}\text { PS3 } & 67 & 69 & 56 & 3 & 69 & 54 & 44 & 41 & 9 & 42 & 28 & 45 & 27 & 67 & 63 & 54 & 14 & 24 & 29 & 34 & 67 & 6\end{array}$

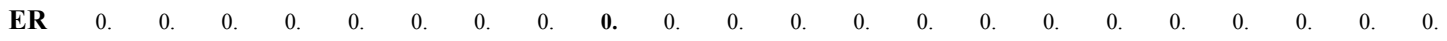

$\begin{array}{lllllllllllllllllllllllllll}\text { PS4 } & 71 & 72 & 65 & 43 & 76 & 6 & 52 & 5 & 9 & 54 & 36 & 5 & 34 & 74 & 73 & 52 & 22 & 25 & 31 & 37 & 72 & 63\end{array}$

$\begin{array}{llllllllllllllllllllllll}\text { ER } & 0 . & 0 . & 0 . & 0 . & 0 . & 0 . & 0 . & 0 . & \mathbf{0 .} & 0 . & 0 . & 0 . & 0 . & 0 . & 0 . & 0 . & 0 . & 0 . & 0 . & 0 . & 0 . & 0 .\end{array}$

$\begin{array}{llllllllllllllllllllllllllll}\text { PS5 } & 68 & 74 & 54 & 39 & 76 & 56 & 48 & 42 & 9 & 57 & 25 & 5 & 3 & 66 & 64 & 55 & 11 & 25 & 3 & 35 & 65 & 6\end{array}$

$\begin{array}{lllllllllllllllllllllll}\text { ER } & 0 . & 0 . & 0 . & 0 . & 0 . & 0 . & 0 . & 0 . & 0 . & \mathbf{0 .} & 0 . & 0 . & 0 . & 0 . & 0 . & 0 . & 0 . & 0 . & 0 . & 0 . & 0 . & 0 .\end{array}$

$\begin{array}{lllllllllllllllllllllllllll}\text { PT1 } & 38 & 47 & 67 & 69 & 48 & 71 & 67 & 38 & 46 & \mathbf{8} & 28 & 39 & 28 & 42 & 51 & 3 & 2 & 17 & 28 & 38 & 48 & 48\end{array}$

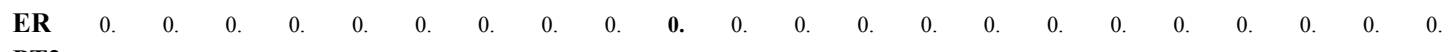

$\begin{array}{lllllllllllllllllllllllll}\text { PT2 } & 37 & 43 & 59 & 59 & 47 & 53 & 58 & 36 & 41 & \mathbf{8} & 41 & 47 & 33 & 45 & 4 & 21 & 3 & 15 & 31 & 3 & 34 & 27\end{array}$

ERPT

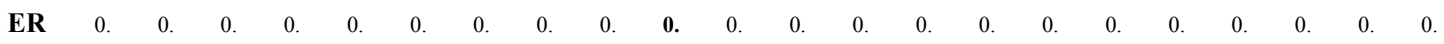

$\begin{array}{llllllllllllllllllllllllll}\text { PT3 } & 43 & 46 & 59 & 62 & 5 & 57 & 61 & 36 & 49 & \mathbf{8} & 38 & 41 & 3 & 46 & 47 & 22 & 26 & 12 & 29 & 33 & 41 & 32\end{array}$

$\begin{array}{lllllllllllllllllllllllll}\text { ER } & 0 . & 0 . & 0 . & 0 . & 0 . & 0 . & 0 . & 0 . & 0 . & \mathbf{0 .} & 0 . & 0 . & 0 . & 0 . & 0 . & 0 . & 0 . & 0 . & 0 . & 0 . & 0 . & 0 .\end{array}$

$\begin{array}{lllllllllllllllllllllllll}\text { PT4 } & 41 & 41 & 68 & 68 & 44 & 68 & 72 & 38 & 48 & \mathbf{8} & 24 & 31 & 16 & 43 & 49 & 23 & 22 & 16 & 19 & 34 & 46 & 38\end{array}$

$\begin{array}{llllllllllllllllllllllll}\text { ER } & 0 . & 0 . & 0 . & 0 . & 0 . & 0 . & 0 . & 0 . & 0 . & \mathbf{0 .} & 0 . & 0 . & 0 . & 0 . & 0 . & 0 . & 0 . & 0 . & 0 . & 0 . & 0 . & 0 .\end{array}$

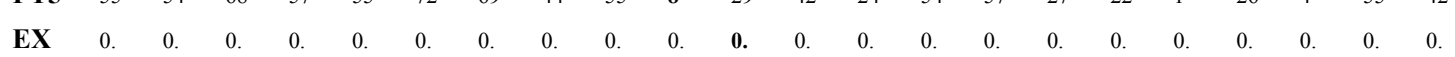

$\begin{array}{llllllllllllllllllllllll}\mathbf{C 1} & 39 & 42 & 36 & 33 & 51 & 33 & 29 & 48 & 38 & 33 & \mathbf{9} & 58 & 61 & 39 & 44 & 37 & 68 & 41 & 45 & 55 & 41 & 54\end{array}$

$\begin{array}{lllllllllllllllllllllllll}\mathbf{E X X} & \mathbf{E X} & 0 . & 0 . & 0 . & 0 . & 0 . & 0 . & 0 . & 0 . & 0 . & 0 . & 1 & 0 . & 0 . & 0 . & 0 . & 0 . & 0 . & 0 . & 0 . & 0 . & 0 . & 0 . \\ & \mathbf{C 2} & 42 & 47 & 42 & 41 & 51 & 39 & 36 & 48 & 37 & 38 & 1 & 66 & 67 & 39 & 49 & 39 & 72 & 49 & 48 & 62 & 42 & 5\end{array}$

$\begin{array}{lllllllllllllllllllllllll}\mathbf{E X} & 0 . & 0 . & 0 . & 0 . & 0 . & 0 . & 0 . & 0 . & 0 . & 0 . & \mathbf{0 .} & 0 . & 0 . & 0 . & 0 . & 0 . & 0 . & 0 . & 0 . & 0 . & 0 . & 0 .\end{array}$

$\begin{array}{lllllllllllllllllllllllll}\text { C3 } & 41 & 4 & 46 & 38 & 41 & 36 & 3 & 49 & 37 & 38 & \mathbf{9} & 71 & 73 & 4 & 46 & 38 & 62 & 4 & 48 & 55 & 36 & 44\end{array}$

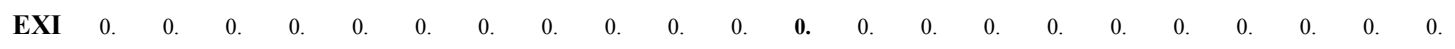

$\begin{array}{llllllllllllllllllllllllll}1 & 46 & 54 & 52 & 44 & 49 & 52 & 37 & 61 & 47 & 46 & 58 & 9 & 71 & 59 & 53 & 49 & 57 & 5 & 71 & 62 & 49 & 51\end{array}$

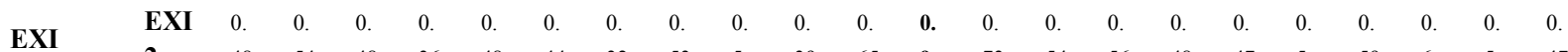
$\begin{array}{lllllllllllllllllllllllll}2 & 48 & 54 & 48 & 36 & 48 & 44 & 32 & 53 & 5 & 39 & 65 & \mathbf{9} & 73 & 54 & 56 & 48 & 47 & 5 & 59 & 6 & 5 & 47\end{array}$ $\begin{array}{lllllllllllllllllllllll}\text { EXI } & 0 . & 0 . & 0 . & 0 . & 0 . & 0 . & 0 . & 0 . & 0 . & 0 . & 0 . & \mathbf{0 .} & 0 . & 0 . & 0 . & 0 . & 0 . & 0 . & 0 . & 0 . & 0 . & 0 .\end{array}$ $\begin{array}{lllllllllllllllllllllllllllll}3 & 51 & 62 & 53 & 46 & 49 & 49 & 41 & 55 & 48 & 47 & 65 & \mathbf{9} & 71 & 57 & 58 & 46 & 46 & 44 & 56 & 57 & 48 & 49\end{array}$ $\begin{array}{llllllllllllllllllllllll}\mathbf{E X} & 0 . & 0 . & 0 . & 0 . & 0 . & 0 . & 0 . & 0 . & 0 . & 0 . & 0 . & 0 . & \mathbf{0 .} & 0 . & 0 . & 0 . & 0 . & 0 . & 0 . & 0 . & 0 . & 0 .\end{array}$ $\begin{array}{llllllllllllllllllllllll}\mathbf{P 1} & 32 & 49 & 34 & 44 & 32 & 37 & 3 & 57 & 27 & 28 & 53 & 78 & \mathbf{9} & 45 & 52 & 53 & 42 & 58 & 65 & 55 & 46 & 46\end{array}$ $\begin{array}{lllllllllllllllllllllll}\text { EX } & 0 . & 0 . & 0 . & 0 . & 0 . & 0 . & 0 . & 0 . & 0 . & 0 . & 0 . & 0 . & \mathbf{0 .} & 0 . & 0 . & 0 . & 0 . & 0 . & 0 . & 0 . & 0 . & 0 . \\ \mathbf{P 2} & 34 & 48 & 31 & 41 & 39 & 34 & 34 & 56 & 28 & 29 & 68 & 73 & \mathbf{9} & 42 & 51 & 52 & 54 & 59 & 6 & 54 & 44 & 5\end{array}$

EXP

\begin{tabular}{llllllllllllllllllllllll} 
EX & 0. & 0. & 0. & 0. & 0. & 0. & 0. & 0. & 0. & 0. & 0. & 0. & $\mathbf{0 .}$ & 0. & 0. & 0. & 0. & 0. & 0. & 0. & 0. & 0. \\
$\mathbf{P 3}$ & 21 & 32 & 2 & 33 & 36 & 2 & 16 & 53 & 27 & 21 & 67 & 6 & $\mathbf{8}$ & 23 & 4 & 54 & 57 & 58 & 38 & 54 & 32 & 44 \\
$\mathbf{E X}$ & 0. & 0. & 0. & 0. & 0. & 0. & 0. & 0. & 0. & 0. & 0. & 0. & $\mathbf{0 .}$ & 0. & 0. & 0. & 0. & 0. & 0. & 0. & 0. & 0. & 0. \\
$\mathbf{P 4}$ & 26 & 38 & 34 & 43 & 36 & 31 & 28 & 56 & 27 & 28 & 51 & 53 & $\mathbf{8}$ & 37 & 44 & 63 & 52 & 32 & 33 & 44 & 37 & 53 \\
\hline
\end{tabular}




\section{Constr} uct

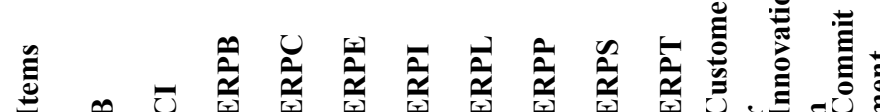

$\begin{array}{llll}\text { HR } & 0 . & 0 .\end{array}$

$\begin{array}{llllllll}\text { E1 } & 66 & 71 & 52 & 24 & 58 & 48 & 34\end{array}$

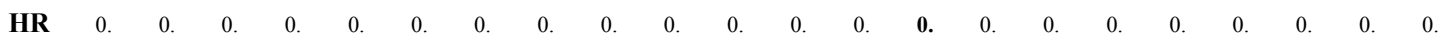

$\begin{array}{lllllllllllllllllllllll}\mathbf{E 2} & 68 & 78 & 64 & 54 & 67 & 6 & 55 & 52 & 74 & 51 & 46 & 58 & 46 & \mathbf{8} & 72 & 57 & 34 & 29 & 35 & 34 & 65 & 56\end{array}$

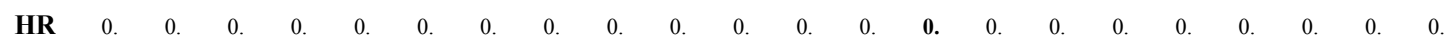

$\begin{array}{llllllllllllllllllllllll}\mathbf{E 3} & 6 & 74 & 5 & 43 & 58 & 54 & 47 & 56 & 63 & 45 & 29 & 44 & 43 & \mathbf{8} & 81 & 46 & 28 & 34 & 43 & 4 & 74 & 52\end{array}$

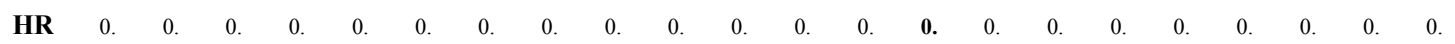

$\begin{array}{lllllllllllllllllllllll}\text { I1 } & 68 & 67 & 48 & 34 & 63 & 37 & 36 & 58 & 65 & 37 & 41 & 61 & 42 & \mathbf{8} & 61 & 52 & 34 & 25 & 55 & 36 & 61 & 58\end{array}$

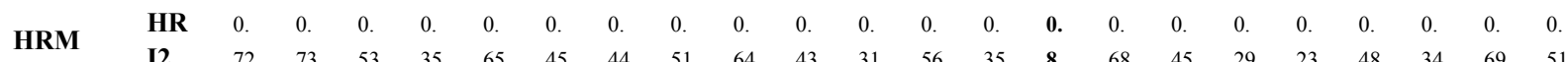

$\begin{array}{lllllllllllllllllllllll}\text { I2 } & 72 & 73 & 53 & 35 & 65 & 45 & 44 & 51 & 64 & 43 & 31 & 56 & 35 & \mathbf{8} & 68 & 45 & 29 & 23 & 48 & 34 & 69 & 51\end{array}$

$\begin{array}{lllllllllllllllllllllll}\text { HR } & 0 . & 0 . & 0 . & 0 . & 0 . & 0 . & 0 . & 0 . & 0 . & 0 . & 0 . & 0 . & 0 . & \mathbf{0 .} & 0 . & 0 . & 0 . & 0 . & 0 . & 0 . & 0 . & 0 . \\ \mathbf{I 3} & 78 & 76 & 62 & 43 & 61 & 61 & 55 & 49 & 68 & 52 & 36 & 57 & 4 & \mathbf{9} & 75 & 48 & 35 & 21 & 5 & 37 & 7 & 55\end{array}$

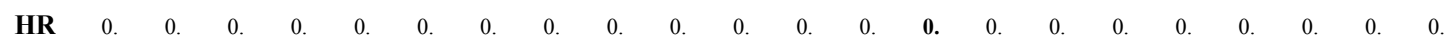

$\begin{array}{lllllllllllllllllllllll}\text { T2 } & 56 & 64 & 42 & 28 & 58 & 37 & 28 & 35 & 54 & 45 & 3 & 44 & 27 & \mathbf{8} & 66 & 28 & 25 & 26 & 5 & 32 & 63 & 41\end{array}$

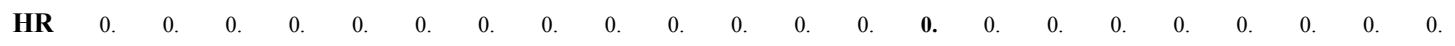

$\begin{array}{lllllllllllllllllllllll}\text { T3 } & 67 & 71 & 63 & 38 & 62 & 49 & 49 & 41 & 63 & 5 & 36 & 52 & 32 & 9 & 69 & 37 & 32 & 2 & 47 & 32 & 65 & 42\end{array}$

$\begin{array}{lllllllllllllllllllllll}\text { HR } & 0 . & 0 . & 0 . & 0 . & 0 . & 0 . & 0 . & 0 . & 0 . & 0 . & 0 . & 0 . & 0 . & \mathbf{0 .} & 0 . & 0 . & 0 . & 0 . & 0 . & 0 . & 0 . & 0 .\end{array}$

$\begin{array}{lllllllllllllllllllllll}\text { TI } & 71 & 74 & 58 & 4 & 54 & 52 & 52 & 33 & 62 & 53 & 36 & 49 & 31 & 9 & 7 & 34 & 24 & 15 & 44 & 23 & 64 & 46\end{array}$

$\begin{array}{lllllllllllllllllllllll}\text { IA1 } & 0 . & 0 . & 0 . & 0 . & 0 . & 0 . & 0 . & 0 . & 0 . & 0 . & 0 . & 0 . & 0 . & 0 . & \mathbf{0 .} & 0 . & 0 . & 0 . & 0 . & 0 . & 0 . & 0 . \\ & 56 & 68 & 51 & 49 & 54 & 54 & 4 & 55 & 56 & 56 & 52 & 58 & 57 & 67 & \mathbf{8} & 46 & 39 & 35 & 56 & 53 & 67 & 54\end{array}$

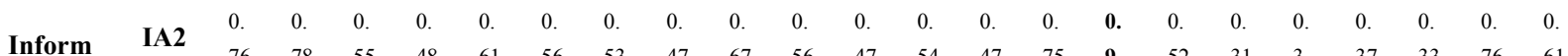

$\begin{array}{llllllllllllllllllllllll} & 76 & 78 & 55 & 48 & 61 & 56 & 53 & 47 & 67 & 56 & 47 & 54 & 47 & 75 & 9 & 52 & 31 & 3 & 37 & 33 & 76 & 61\end{array}$

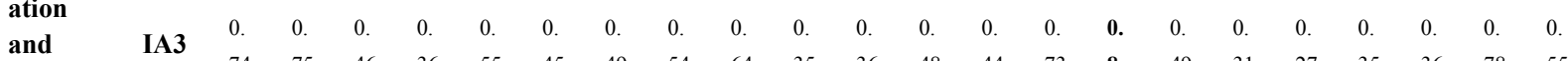
$\begin{array}{llllllllllllllllllllllll}\text { Analysi } & 74 & 75 & 46 & 36 & 55 & 45 & 49 & 54 & 64 & 35 & 36 & 48 & 44 & 73 & \mathbf{8} & 49 & 31 & 27 & 35 & 36 & 78 & 55\end{array}$

IA4 $\begin{array}{llllllllllllllllllllll}0 . & 0 . & 0 . & 0 . & 0 . & 0 . & 0 . & 0 . & 0 . & 0 . & 0 . & 0 . & 0 . & 0 . & \mathbf{0 .} & 0 . & 0 . & 0 . & 0 . & 0 . & 0 . & 0 . \\ \end{array}$

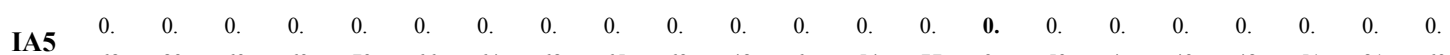

$\begin{array}{llllllllllllllllllllllllll}\text { IA5 } & 68 & 83 & 63 & 63 & 73 & 66 & 64 & 68 & 65 & 62 & 48 & 6 & 54 & 77 & 9 & 53 & 4 & 43 & 48 & 51 & 81 & 63\end{array}$

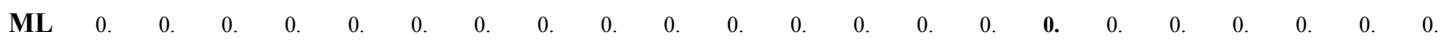

$\begin{array}{llllllllllllllllllllllll}1 & 53 & 68 & 36 & 46 & 67 & 41 & 39 & 59 & 6 & 33 & 34 & 54 & 6 & 53 & 59 & 9 & 34 & 47 & 36 & 48 & 62 & 73\end{array}$

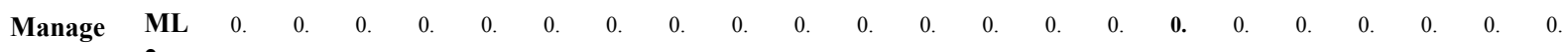
$\begin{array}{lllllllllllllllllllllllllll}\text { ment } & 2 & 48 & 53 & 21 & 32 & 54 & 27 & 26 & 52 & 47 & 14 & 29 & 43 & 53 & 41 & 46 & \mathbf{9} & 3 & 53 & 32 & 41 & 49 & 72\end{array}$

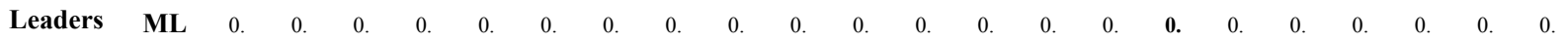
$\begin{array}{llllllllllllllllllllllllll}\text { hip } & 3 & 48 & 58 & 36 & 51 & 66 & 38 & 41 & 62 & 53 & 34 & 44 & 5 & 65 & 45 & 51 & \mathbf{9} & 46 & 47 & 27 & 44 & 51 & 75\end{array}$

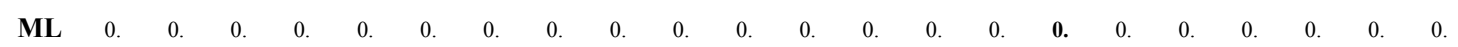
$\begin{array}{lllllllllllllllllllllllllll}4 & 46 & 6 & 35 & 5 & 63 & 37 & 4 & 6 & 5 & 29 & 4 & 47 & 62 & 48 & 53 & 9 & 44 & 49 & 24 & 39 & 49 & 72\end{array}$ $\begin{array}{lllllllllllllllllllllll}\mathbf{O P} & 0 . & 0 . & 0 . & 0 . & 0 . & 0 . & 0 . & 0 . & 0 . & 0 . & 0 . & 0 . & 0 . & 0 . & 0 . & 0 . & \mathbf{0 .} & 0 . & 0 . & 0 . & 0 . & 0 .\end{array}$ $\begin{array}{llllllllllllllllllllllll}\text { C4 } & 19 & 25 & 33 & 43 & 37 & 27 & 33 & 47 & 16 & 19 & 61 & 44 & 59 & 18 & 28 & 43 & \mathbf{8} & 41 & 23 & 46 & 21 & 41\end{array}$ $\begin{array}{lllllllllllllllllllllll}\mathbf{O P} & 0 . & 0 . & 0 . & 0 . & 0 . & 0 . & 0 . & 0 . & 0 . & 0 . & 0 . & 0 . & 0 . & 0 . & 0 . & 0 . & \mathbf{0 .} & 0 . & 0 . & 0 . & 0 . & 0 .\end{array}$ $\begin{array}{llllllllllllllllllllllll}\text { Custom } & \text { C5 } & 11 & 14 & 3 & 29 & 22 & 25 & 22 & 45 & 06 & 11 & 48 & 4 & 51 & 18 & 17 & 33 & \mathbf{8} & 31 & 39 & 53 & 17 & 37\end{array}$

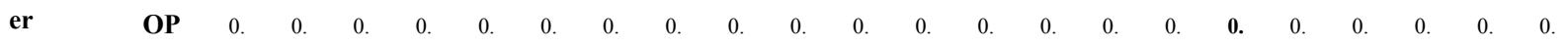
$\begin{array}{lllllllllllllllllllllll}\mathbf{C 6} & 39 & 33 & 37 & 21 & 44 & 32 & 29 & 31 & 28 & 3 & 62 & 49 & 42 & 29 & 3 & 26 & \mathbf{8} & 34 & 44 & 62 & 33 & 41\end{array}$

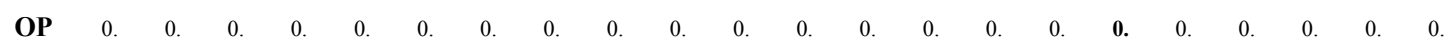
$\begin{array}{lllllllllllllllllllllll}\text { C7 } & 35 & 4 & 35 & 33 & 43 & 33 & 26 & 48 & 36 & 32 & 61 & 45 & 46 & 43 & 42 & 35 & \mathbf{8} & 51 & 49 & 63 & 37 & 4\end{array}$ $\begin{array}{lllllllllllllllllllllll}\mathbf{O P} & 0 . & 0 . & 0 . & 0 . & 0 . & 0 . & 0 . & 0 . & 0 . & 0 . & 0 . & 0 . & 0 . & 0 . & 0 . & 0 . & 0 . & \mathbf{0 .} & 0 . & 0 . & 0 . & 0 .\end{array}$ $\begin{array}{lllllllllllllllllllllll}\text { F1 } & 13 & 34 & 15 & 33 & 32 & 19 & 18 & 46 & 18 & 12 & 49 & 48 & 63 & 23 & 36 & 52 & 57 & 9 & 53 & 57 & 32 & 45\end{array}$

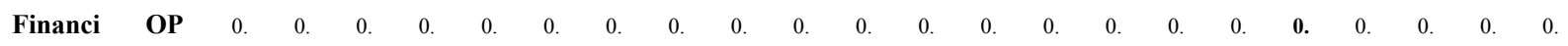
$\begin{array}{llllllllllllllllllllllll}\text { al } & \text { F2 } & 06 & 22 & 16 & 29 & 29 & 19 & 23 & 44 & 2 & 15 & 24 & 37 & 37 & 18 & 23 & 3 & 2 & 7 & 28 & 24 & 23 & 23\end{array}$

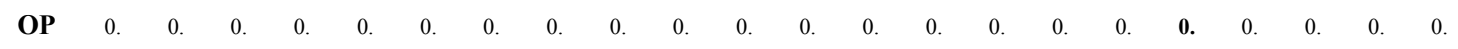
$\begin{array}{lllllllllllllllllllllllllll}\text { F3 } & 24 & 31 & 12 & 35 & 34 & 18 & 26 & 47 & 24 & 16 & 3 & 39 & 41 & 25 & 33 & 39 & 29 & \mathbf{8} & 27 & 35 & 29 & 32\end{array}$

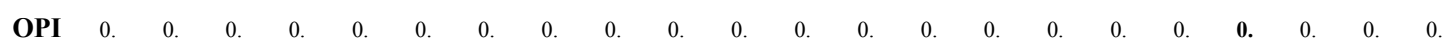
$\begin{array}{lllllllllllllllllllllllll}\text { Internal } & \mathbf{1 0} & 25 & 34 & 17 & 11 & 34 & 19 & 05 & 34 & 21 & 26 & 47 & 5 & 48 & 4 & 36 & 21 & 35 & 39 & \mathbf{8} & 48 & 35 & 4\end{array}$ \begin{tabular}{llllllllllllllllllllllll} 
Process & OPI & 0. & 0. & 0. & 0. & 0. & 0. & 0. & 0. & 0. & 0. & 0. & 0. & 0. & 0. & 0. & 0. & 0. & 0. & $\mathbf{0 .}$ & 0. & 0. & 0. \\
& $\mathbf{1 1}$ & 26 & 44 & 37 & 3 & 42 & 36 & 27 & 4 & 34 & 36 & 37 & 61 & 43 & 45 & 36 & 29 & 33 & 48 & 7 & 55 & 35 & 32 \\
\hline
\end{tabular} 


\begin{tabular}{|c|c|c|c|c|c|c|c|c|c|c|c|c|c|c|c|c|c|c|c|c|c|c|c|}
\hline $\begin{array}{l}\text { Constr } \\
\text { uct }\end{array}$ & E气 & $\oplus$ & ర & $\frac{n}{a}$ & $\begin{array}{l}\cup \\
\frac{1}{n}\end{array}$ & $\frac{0}{20}$ & $\underline{a}$ & $\frac{a}{a}$ & 술 & $\underline{\tilde{a}}$ & $\frac{1}{\alpha}$ & 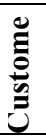 & 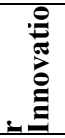 & =نٍ & $\underline{\underline{x}}$ & $\Xi$ & $\sum$ & ○ & $\frac{1}{0}$ & $\overline{0}$ & $\frac{1}{0}$ & $\theta$ & $\hat{\sigma}$ \\
\hline \multirow{14}{*}{$\begin{array}{l}\text { Learnin } \\
\text { g and } \\
\text { Growth }\end{array}$} & OPI & 0. & 0. & 0. & 0. & 0. & 0. & 0. & 0. & 0. & 0. & 0. & 0. & 0. & 0. & 0. & 0. & 0. & 0. & 0. & 0. & 0. & 0. \\
\hline & 8 & 22 & 35 & 27 & 17 & 26 & 29 & 14 & 3 & 22 & 19 & 29 & 48 & 38 & 41 & 38 & 17 & 44 & 37 & 8 & 56 & 4 & 29 \\
\hline & OPI & 0. & 0. & 0. & 0. & 0. & 0. & 0. & 0 . & 0. & 0. & 0 . & 0 . & 0. & 0 . & 0. & 0. & 0. & 0. & 0. & 0. & 0. & 0. \\
\hline & 9 & 34 & 46 & 31 & 22 & 29 & 31 & 17 & 33 & 32 & 22 & 45 & 6 & 59 & 5 & 43 & 36 & 43 & 3 & 8 & 47 & 41 & 5 \\
\hline & OP & 0. & 0. & 0. & 0. & 0. & 0. & 0. & 0. & 0. & 0. & 0 . & 0 . & 0. & 0. & 0. & 0. & 0. & 0. & 0. & 0. & 0. & 0. \\
\hline & L12 & 31 & 42 & 51 & 46 & 48 & 47 & 43 & 55 & 43 & 48 & 57 & 54 & 48 & 31 & 4 & 38 & 59 & 48 & 52 & 8 & 43 & 44 \\
\hline & OP & 0. & 0. & 0. & 0. & 0. & 0. & 0. & 0. & 0. & 0. & 0 . & 0 . & 0. & 0. & 0. & 0. & 0. & 0. & 0. & 0. & 0. & 0. \\
\hline & L13 & 18 & 27 & 26 & 28 & 3 & 29 & 21 & 49 & 2 & 26 & 43 & 49 & 51 & 27 & 33 & 34 & 59 & 49 & 54 & 8 & 36 & 32 \\
\hline & OP & 0. & 0. & 0. & 0. & 0. & 0. & 0. & 0. & 0. & 0. & 0 . & 0 . & 0. & 0. & 0. & 0. & 0. & 0. & 0. & 0. & 0. & 0. \\
\hline & L14 & 51 & 47 & 48 & 31 & 47 & 57 & 44 & 4 & 44 & 44 & 42 & 51 & 37 & 42 & 46 & 33 & 46 & 17 & 45 & 8 & 48 & 44 \\
\hline & OP & 0. & 0. & 0. & 0. & 0. & 0. & 0. & 0. & 0. & 0. & 0 . & 0 . & 0. & 0. & 0. & 0. & 0. & 0. & 0. & 0. & 0. & 0. \\
\hline & L15 & 31 & 41 & 29 & 21 & 44 & 35 & 24 & 45 & 35 & 23 & 53 & 57 & 59 & 29 & 4 & 45 & 59 & 5 & 58 & 9 & 44 & 45 \\
\hline & \multirow{2}{*}{ SD1 } & 0. & 0. & 0. & 0. & 0. & 0. & 0. & 0. & 0. & 0. & 0 . & 0 . & 0. & 0. & 0. & 0. & 0. & 0. & 0. & 0. & 0. & 0. \\
\hline & & 72 & 73 & 55 & 42 & 62 & 59 & 52 & 61 & 69 & 48 & 39 & 51 & 47 & 7 & 82 & 51 & 27 & 28 & 39 & 43 & 9 & 63 \\
\hline \multirow{6}{*}{$\begin{array}{l}\text { Service } \\
\text { Design }\end{array}$} & \multirow{2}{*}{ SD2 } & 0. & 0. & 0. & 0. & 0. & 0. & 0. & 0. & 0. & 0. & 0 . & 0 . & 0. & 0. & 0. & 0. & 0. & 0. & 0. & 0. & 0. & 0. \\
\hline & & 69 & 77 & 59 & 47 & 73 & 6 & 58 & 62 & 69 & 5 & 4 & 49 & 41 & 71 & 79 & 49 & 36 & 32 & 42 & 52 & 9 & 6 \\
\hline & \multirow{2}{*}{ SD3 } & 0. & 0. & 0. & 0. & 0. & 0. & 0. & 0. & 0. & 0. & 0 . & 0 . & 0. & 0. & 0. & 0. & 0. & 0. & 0. & 0. & 0. & 0. \\
\hline & & 72 & 78 & 55 & 47 & 74 & 56 & 5 & 58 & 71 & 52 & 37 & 49 & 42 & 77 & 78 & 57 & 3 & 38 & 49 & 49 & 9 & 61 \\
\hline & SP1 & 0. & 0. & 0. & 0. & 0. & 0. & 0. & 0. & 0. & 0. & 0 . & 0 . & 0. & 0. & 0. & 0. & 0. & 0. & 0. & 0. & 0. & 0. \\
\hline & SFI & 48 & 52 & 3 & 33 & 52 & 39 & 36 & 46 & 48 & 27 & 34 & 36 & 49 & 43 & 5 & 66 & 36 & 35 & 35 & 38 & 53 & 8 \\
\hline \multirow{6}{*}{$\begin{array}{l}\text { Stratgic } \\
\text { Plannin } \\
\mathrm{g}\end{array}$} & \multirow{2}{*}{ SP2 } & 0. & 0. & 0. & 0. & 0. & 0. & 0. & 0. & 0. & 0. & 0 . & 0 . & 0. & 0. & 0. & 0. & 0. & 0. & 0. & 0. & 0. & 0. \\
\hline & & 52 & 62 & 48 & 54 & 69 & 52 & 53 & 49 & 57 & 47 & 39 & 48 & 5 & 49 & 57 & 75 & 41 & 43 & 41 & 45 & 59 & 9 \\
\hline & \multirow{2}{*}{ SP3 } & 0. & 0. & 0. & 0. & 0. & 0. & 0. & 0. & 0. & 0. & 0 . & 0 . & 0. & 0. & 0. & 0. & 0. & 0. & 0. & 0. & 0. & 0. \\
\hline & & 61 & 65 & 56 & 46 & 74 & 6 & 48 & 57 & 69 & 43 & 56 & 57 & 52 & 56 & 58 & 7 & 55 & 32 & 45 & 52 & 62 & 9 \\
\hline & \multirow{2}{*}{ SP4 } & 0. & 0. & 0. & 0. & 0. & 0. & 0. & 0. & 0. & 0. & 0 . & 0 . & 0. & 0. & 0. & 0. & 0. & 0. & 0. & 0. & 0. & 0. \\
\hline & & 61 & 63 & 42 & 46 & 64 & 51 & 43 & 6 & 61 & 42 & 51 & 47 & 48 & 55 & 63 & 64 & 39 & 44 & 42 & 41 & 59 & 8 \\
\hline
\end{tabular}

\subsubsection{The Convergent Validity}

The convergent validity is the degree of a group of items converges to measure a specific variable (Hair et al., 2010). In SEM literature, it can be confirmed by testing the composite reliability, the loading, and the average variance extracted (AVE). To consider the items are highly loaded and statistically significant, factor loading should at least 0.7 , AVE is at least 0.5 , and the composite reliability is at least 0.7 . Table 3 shows that all these criteria have been achieved and confirmed. Therefore, the results of the outer model (measurement model) have suitable convergent validity (Bagozzi \& Yi, 1988).

Table 3 . The convergent validity analysis

\begin{tabular}{|c|c|c|c|c|c|}
\hline Construct & Items & Loadings & Cronbach's Alpha & $\mathbf{C R}^{\mathrm{a}}$ & $\mathbf{A V E} \mathbf{E}^{\mathbf{b}}$ \\
\hline \multirow{3}{*}{ Benchmarking } & B1 & 0.928 & \multirow{3}{*}{0.852} & \multirow{3}{*}{0.917} & \multirow{3}{*}{0.724} \\
\hline & B2 & 0.884 & & & \\
\hline & B3 & 0.877 & & & \\
\hline \multirow{4}{*}{ Continuous Improvement } & CI1 & 0.902 & \multirow{4}{*}{0.915} & \multirow{4}{*}{0.940} & \multirow{4}{*}{0.797} \\
\hline & CI2 & 0.926 & & & \\
\hline & CI3 & 0.867 & & & \\
\hline & CI4 & 0.875 & & & \\
\hline \multirow{5}{*}{ ERP-Business Process Skills } & ERPB1 & 0.851 & \multirow{5}{*}{0.909} & \multirow{5}{*}{0.933} & \multirow{5}{*}{0.736} \\
\hline & ERPB2 & 0.876 & & & \\
\hline & ERPB3 & 0.873 & & & \\
\hline & ERPB4 & 0.919 & & & \\
\hline & ERPB5 & 0.763 & & & \\
\hline ERP-Change Readiness & ERPC1 & 0.861 & 0.942 & 0.956 & 0.812 \\
\hline
\end{tabular}




\begin{tabular}{|c|c|c|c|c|c|}
\hline Construct & Items & Loadings & Cronbach's Alpha & $\mathbf{C R}^{\mathrm{a}}$ & $\mathbf{A V E}^{\mathrm{b}}$ \\
\hline & ERPC2 & 0.932 & & & \\
\hline & ERPC3 & 0.880 & & & \\
\hline & ERPC4 & 0.942 & & & \\
\hline & ERPC5 & 0.888 & & & \\
\hline & ERPE1 & 0.861 & & & \\
\hline & ERPE2 & 0.909 & & & \\
\hline \multirow[t]{5}{*}{ ERP-Executive Commitment } & ERPE3 & 0.925 & 0.915 & 0.938 & 0.752 \\
\hline & ERPE4 & 0.911 & & & \\
\hline & ERPE5 & 0.714 & & & \\
\hline & ERPI1 & 0.840 & & & \\
\hline & ERPI2 & 0.880 & & & \\
\hline \multirow[t]{5}{*}{ ERP-IT Skills } & ERPI3 & 0.891 & 0.922 & 0.941 & 0.763 \\
\hline & ERPI4 & 0.934 & & & \\
\hline & ERPI5 & 0.817 & & & \\
\hline & ERPL1 & 0.778 & & & \\
\hline & ERPL2 & 0.811 & & & \\
\hline \multirow[t]{5}{*}{ ERP-Learning } & ERPL3 & 0.851 & 0.892 & 0.921 & 0.699 \\
\hline & ERPL4 & 0.894 & & & \\
\hline & ERPL5 & 0.842 & & & \\
\hline & ERPP1 & 0.903 & & & \\
\hline & ERPP2 & 0.905 & & & \\
\hline \multirow[t]{5}{*}{ ERP-Project Management } & ERPP3 & 0.895 & 0.920 & 0.941 & 0.761 \\
\hline & ERPP4 & 0.895 & & & \\
\hline & ERPP5 & 0.755 & & & \\
\hline & ERPS1 & 0.840 & & & \\
\hline & ERPS2 & 0.887 & & & \\
\hline \multirow[t]{5}{*}{ ERP-Strategic IT Planning } & ERPS3 & 0.901 & 0.926 & 0.944 & 0.773 \\
\hline & ERPS4 & 0.904 & & & \\
\hline & ERPS5 & 0.862 & & & \\
\hline & ERPT1 & 0.825 & & & \\
\hline & ERPT2 & 0.766 & & & \\
\hline \multirow[t]{4}{*}{ ERP-Training } & ERPT3 & 0.850 & 0.877 & 0.911 & 0.671 \\
\hline & ERPT4 & 0.847 & & & \\
\hline & ERPT5 & 0.805 & & & \\
\hline & EXC1 & 0.927 & & & \\
\hline \multirow[t]{3}{*}{ Excellence-Customer Focus } & EXC2 & 0.951 & 0.930 & 0.956 & 0.878 \\
\hline & EXC3 & 0.933 & & & \\
\hline & EXI1 & 0.884 & & & \\
\hline \multirow[t]{3}{*}{ Excellence-Innovation } & EXI2 & 0.908 & 0.883 & 0.928 & 0.811 \\
\hline & EXI3 & 0.910 & & & \\
\hline & EXP1 & 0.855 & & & \\
\hline \multirow{6}{*}{ Excellence-Personnel Commitment } & EXP2 & 0.862 & 0860 & 0005 & 0704 \\
\hline & EXP3 & 0.837 & 0.800 & 0.905 & 0.104 \\
\hline & EXP4 & 0.802 & & & \\
\hline & HRE1 & 0.862 & & & \\
\hline & HRE2 & 0.785 & & & \\
\hline & HRE3 & 0.785 & & & \\
\hline \multirow[t]{4}{*}{ HRM } & HRI1 & 0.793 & 0.945 & 0.954 & 0.696 \\
\hline & HRI2 & 0.844 & & & \\
\hline & HRI3 & 0.889 & & & \\
\hline & HRT2 & 0.776 & & & \\
\hline
\end{tabular}




\begin{tabular}{|c|c|c|c|c|c|}
\hline Construct & Items & Loadings & Cronbach's Alpha & $\mathbf{C R}^{\mathrm{a}}$ & $\mathbf{A V E} \mathbf{E}^{\mathrm{b}}$ \\
\hline \multirow{7}{*}{ Information and Analysis } & HRT3 & 0.905 & \multirow{8}{*}{0.920} & \multirow{7}{*}{0.940} & \multirow{6}{*}{0.758} \\
\hline & HRTI & 0.860 & & & \\
\hline & IA1 & 0.801 & & & \\
\hline & IA2 & 0.910 & & & \\
\hline & IA3 & 0.848 & & & \\
\hline & IA4 & 0.888 & & & \\
\hline & IA5 & 0.902 & & & \\
\hline \multirow{4}{*}{ Management Leadership } & ML1 & 0.924 & & \multirow{4}{*}{0.956} & \multirow{4}{*}{0.844} \\
\hline & ML2 & 0.892 & \multirow{3}{*}{0.938} & & \\
\hline & ML3 & 0.938 & & & \\
\hline & ML4 & 0.920 & & & \\
\hline \multirow{4}{*}{ Customer } & OPC4 & 0.799 & \multirow{4}{*}{0.823} & \multirow{4}{*}{0.882} & \multirow{5}{*}{0.653} \\
\hline & OPC5 & 0.824 & & & \\
\hline & OPC6 & 0.830 & & & \\
\hline & OPC7 & 0.777 & & & \\
\hline \multirow{3}{*}{ Financial } & OPF1 & 0.866 & \multirow{3}{*}{0.715} & \multirow{3}{*}{0.833} & \\
\hline & OPF2 & 0.740 & & & \multirow[t]{2}{*}{0.625} \\
\hline & OPF3 & 0.762 & & & \\
\hline \multirow{4}{*}{ Internal Process } & OPI10 & 0.794 & \multirow{4}{*}{0.799} & \multirow{4}{*}{0.869} & \multirow{4}{*}{0.625} \\
\hline & OPI11 & 0.719 & & & \\
\hline & OPI8 & 0.822 & & & \\
\hline & OPI9 & 0.823 & & & \\
\hline \multirow{4}{*}{ Learning and Growth } & OPL12 & 0.771 & \multirow{4}{*}{0.813} & \multirow{4}{*}{0.877} & \multirow{4}{*}{0.641} \\
\hline & OPL13 & 0.803 & & & \\
\hline & OPL14 & 0.754 & & & \\
\hline & OPL15 & 0.869 & & & \\
\hline \multirow{3}{*}{ Service Design } & SD1 & 0.892 & \multirow{3}{*}{0.894} & \multirow{3}{*}{0.934} & \multirow{3}{*}{0.826} \\
\hline & SD2 & 0.943 & & & \\
\hline & SD3 & 0.890 & & & \\
\hline \multirow{4}{*}{ Stratgic Planning } & SP1 & 0.841 & & & \\
\hline & SP2 & 0.884 & 0.889 & & \\
\hline & SP3 & 0.894 & 0.889 & 0.923 & 0.751 \\
\hline & SP4 & 0.846 & & & \\
\hline
\end{tabular}

a: $\mathrm{CR}=(\Sigma$ factor loading $) 2 /\{(\Sigma$ factor loading $) 2)+\Sigma$ (variance of error $)\}$

b: AVE $=\Sigma$ (factor loading) $2 /(\Sigma$ (factor loading $) 2+\Sigma($ variance of error $)\}$

\subsubsection{The Discriminant Validity}

The literature of SEM defined the discriminant validity as the degree of items can distinguish a construct from other model's constructs. According to Compeau et al. (1999), items of each construct should have variances among them more than with other constructs in the model. Table 4 shows that the diagonal line of values that contain the square root of AVE and below it there are the correlations of the constructs. To examine the discriminant validity, the values of the diagonal line should be compared with other off diagonal ones. As we can see in the table, the values of the diagonal line are higher than others in their respective columns and rows and therefore confirmed the discriminant validity of the model based on Fornell and Larcker's (1981) criterion. 
Table 4. Correlation and discriminant validity

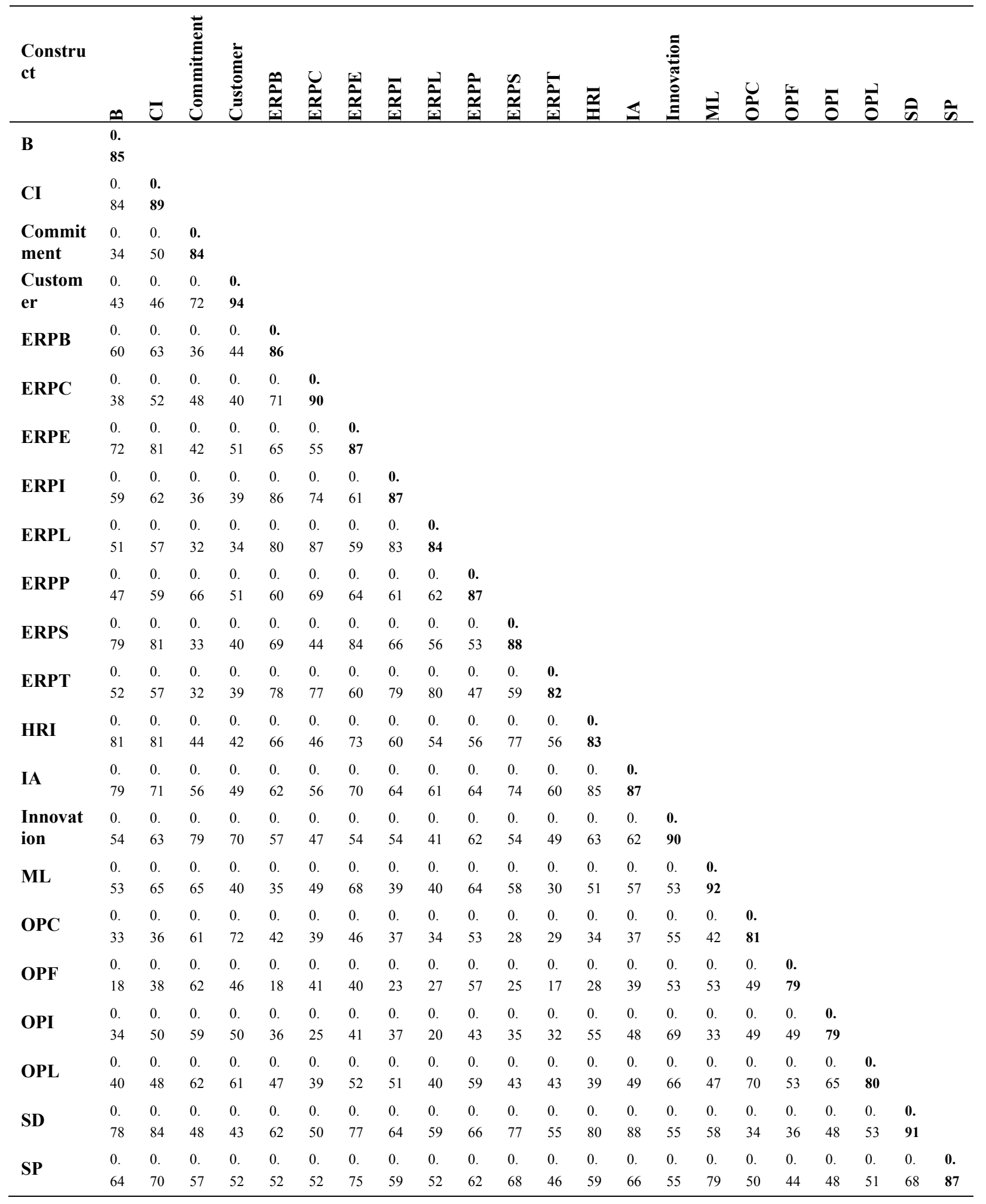

\subsection{The Inner Model (Structural Model), and Hypotheses Testing}

\subsubsection{Testing the Direct Hypotheses}

After testing the validity and reliability of the construct, the next step is to examine in the inner model through hypotheses testing by running Algorithm and Bootstrapping in PLS. Figure 2 and Table 5 illustrated the results. 


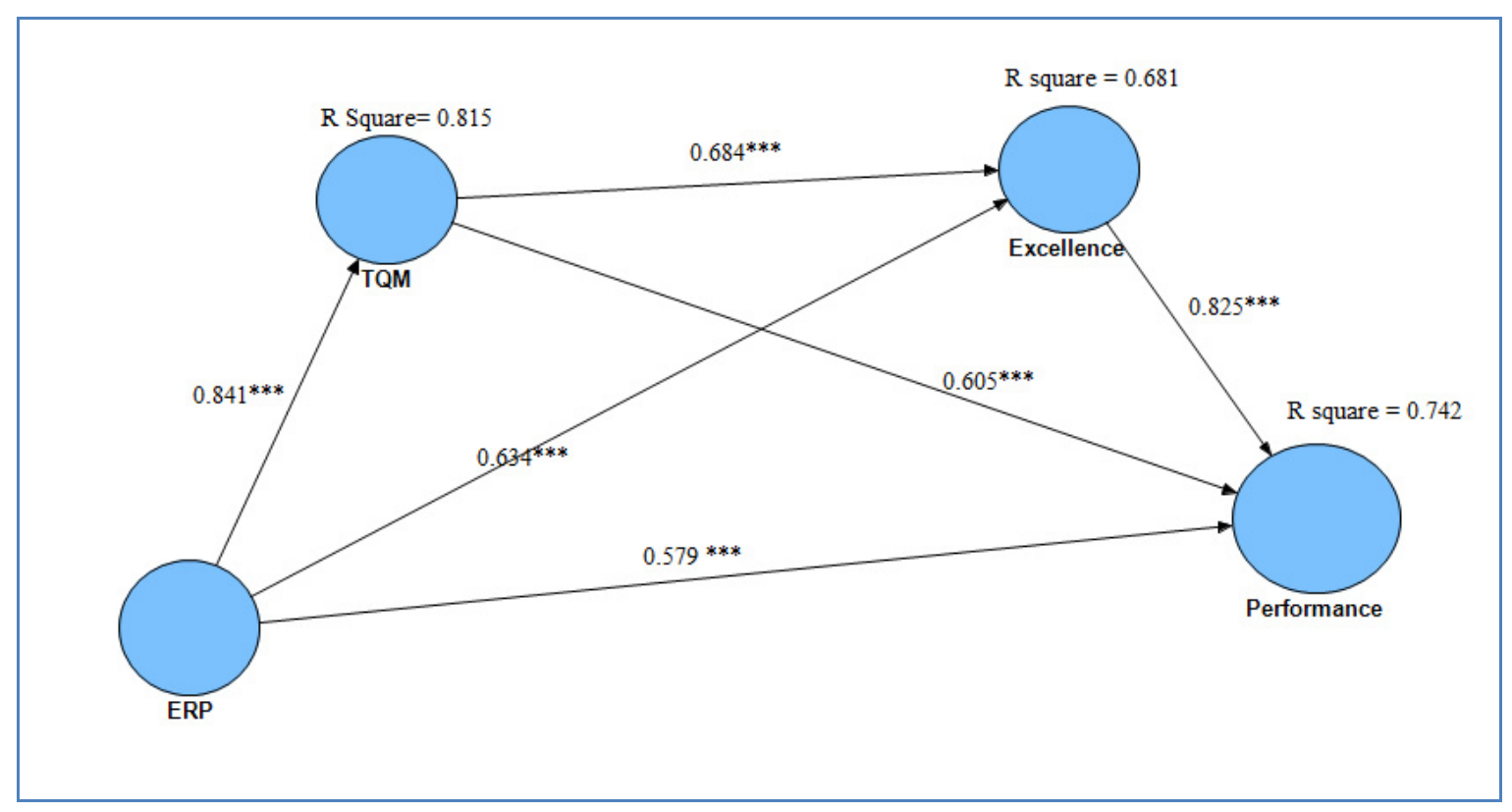

Figure 2. Hypotheses testing results

Table 5. Hypotheses testing results

\begin{tabular}{lllllll}
\hline No & Hypothesis & Path Coefficient & Standard Error & T Value & P Value & Decision \\
\hline H1 & ERP-> Performance & $0.579 * * *$ & 0.038 & 15.293 & 0.000 & Supported \\
H2 & ERP-> TQM & $0.841^{* * *}$ & 0.019 & 44.301 & 0.000 & Supported \\
H3 & ERP-> Excellence & $0.634 * * *$ & 0.041 & 15.337 & 0.000 & Supported \\
H4 & TQM-> Performance & $0.605 * * *$ & 0.047 & 12.973 & 0.000 & Supported \\
H5 & TQM-> Excellence & $0.684 * * *$ & 0.047 & 14.422 & 0.000 & Supported \\
H6 & Excellence-> Performance & $0.825 * * *$ & 0.024 & 34.747 & 0.000 & Supported \\
\hline
\end{tabular}

$* * *: \mathrm{p}<0.001 ; * *: \mathrm{p}<0.01 ; *: \mathrm{P}<0.05$

Figure 2 and Table 5 show that all the six hypotheses have positive and significant results at the 0.001 level of significance $(\beta=0.579, \mathrm{t}=15.293, \mathrm{p}<0.001),(\beta=0.841, \mathrm{t}=44.301, \mathrm{p}<0.001),(\beta=0.634, \mathrm{t}=15.337, \mathrm{p}<0.001)$, $(\beta=0.605, \mathrm{t}=12.973, \mathrm{p}<0.001),(\beta=0.684, \mathrm{t}=14.422, \mathrm{p}<0.001)$, and $(\beta=0.825, \mathrm{t}=34.747, \mathrm{p}<0.001)$ respectively. Therefore, these results supported the hypotheses of the study H1, H2, H3, H4, H5, and H6.

4.2.2 Testing the Mediation Role of TQM and Organizational Excellence

In order to examine the mediation effect of TQM and organizational excellence, SmartPLS was used to estimate the indirect impact between variables. Table 6 shows that TQM has a mediation effect on the relationship between ERP and organizational performance at the 0.001 level of significance $(\beta=0.199, \mathrm{t}=2.695, \mathrm{p}<0.001)$ and therefore confirm H7. The other hypothesis (H8) also confirmed where organizational excellence was found to have a mediation effect on the relationship between ERP and organizational performance at the 0.001 level of significance $(\beta=0.491, \mathrm{t}=12.332, \mathrm{p}<0.001)$. To decide whether these medications are full or partial, Variance Accounted For (VAF) has been employed. According to VAF (58\%), TQM was found to have a partial mediation whereas organizational excellence has full mediation (81\%) (Hair et al., 2014). 
Table 6. Mediation analysis results

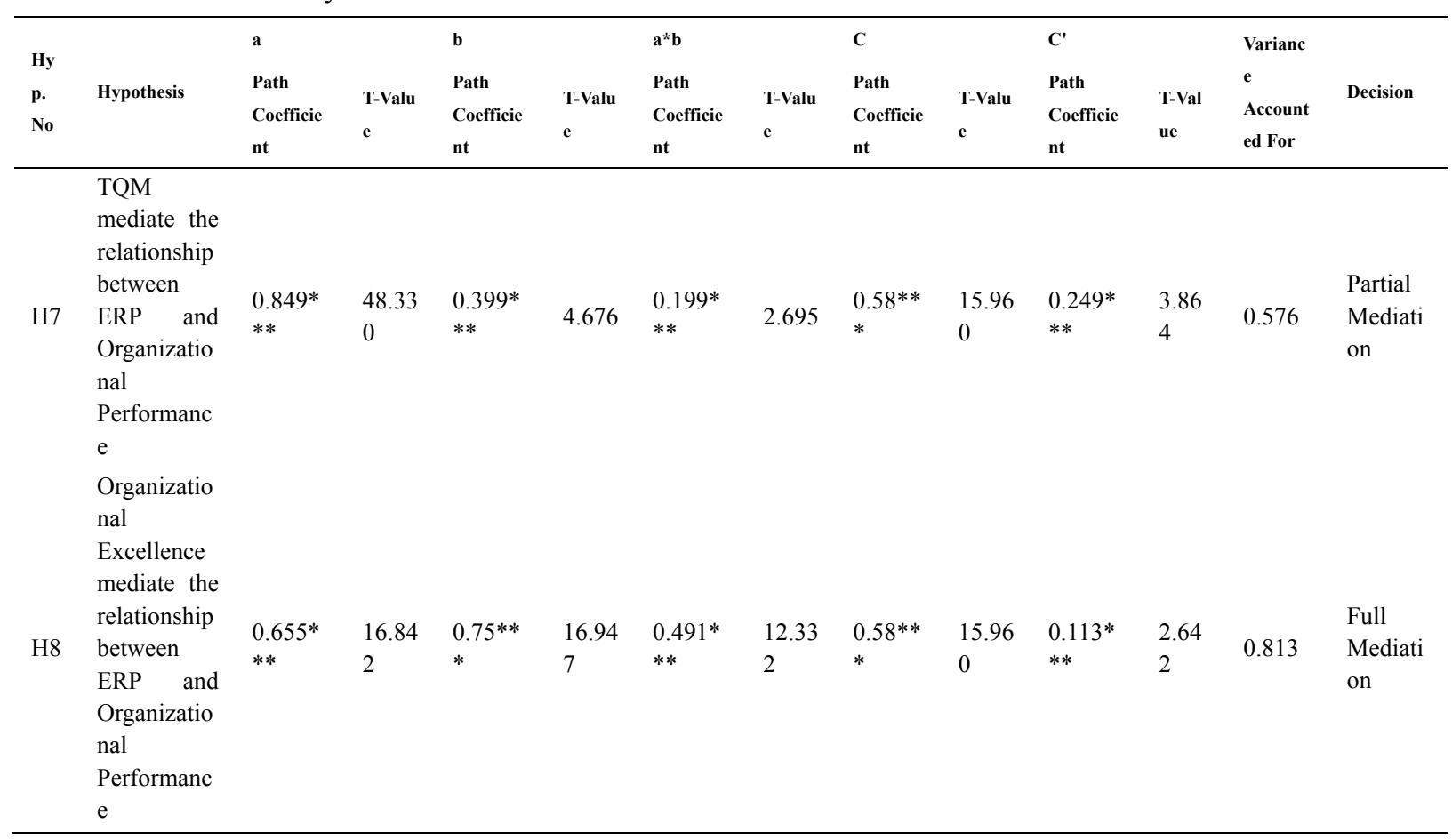

\subsection{Predictive Relevance of the Model}

R-square, cross-validated redundancy and cross-validated communality were employed to test the predictive power of the model. According to Cohen (1988), values of R-square are substantial with 0.26, moderate with 0.13 , and weak with 0.02 . All values in the table of R-square are considered substantial. Cross-validated redundancy and cross-validated communality are the medium to assess the model's quality. To extract them, blindfolding procedure in PLS was employed. Their values should be more than zero to say that the model has predictive quality (Fornell \& Cha, 1994). Table 7 shows values more than zero and therefore confirmed that the model has prediction quality.

Table 7. Prediction relevance of the model

\begin{tabular}{llll}
\hline Construct & R Square & $\begin{array}{l}\text { Cross-validity } \\
\text { Redundancy }\end{array}$ & $\begin{array}{l}\text { Cross-validity } \\
\text { Communality }\end{array}$ \\
\hline Total Quality Management & 0.735 & 0.407 & 0.582 \\
Organizational Excellence & 0.505 & 0.309 & 0.642 \\
Organizational performance & 0.687 & 0.292 & 0.430 \\
\hline
\end{tabular}

\subsection{Goodness of Fit (GoF) of the Model}

According to Wetzels et al. (2009), GoF can be confirmed based on the criteria: (small $=0.1$, medium $=0.25$, large $=0.36$ ). Table 8 below shows that the GoF value was 0.743 which considered large value.

Table 8 .

\begin{tabular}{llll}
\hline Construct & R Square & Average Variance Extracted & Goodness of Fit \\
\hline Benchmarking & 0.731 & 0.724 & \\
Continuous Improvement & 0.877 & 0.797 & \\
ERPB & & 0.736 \\
ERPC & & 0.812 \\
ERPE & & 0.752 & \\
\hline
\end{tabular}




\begin{tabular}{llll}
\hline Construct & R Square & Average Variance Extracted & Goodness of Fit \\
\hline ERPI & & 0.763 & \\
ERPL & & 0.699 & \\
ERPP & & 0.761 & \\
ERPS & & 0.773 & \\
ERPT & & 0.671 & \\
EXC & 0.758 & 0.878 & \\
EXI & 0.860 & 0.811 & \\
EXP & 0.832 & 0.704 & \\
HRM & 0.774 & 0.696 & \\
Information and Analysis & 0.848 & 0.758 & \\
Management Leadership & 0.572 & 0.844 & \\
Customer & 0.703 & 0.653 & \\
Financial & 0.508 & 0.625 & \\
Internal Process & 0.655 & 0.625 & \\
Learning and Growth & 0.814 & 0.641 & \\
Service Design & 0.811 & 0.826 & \\
Strategic Planning & 0.700 & 0.751 & \\
Average & $\mathbf{0 . 7 4 6}$ & $\mathbf{0 . 7 4 1}$ & \\
\hline
\end{tabular}

\section{Discussion and Conclusion}

The main purpose of this study is to examine the direct and indirect effect of ERP on organizational performance. Due to the inconsistency in the literature of the effect of ERP on organizational performance, two mediators' variables have been proposed as mechanisms to explain this relationship in different context. These two variables are TQM and organizational excellence. As expected from the nature and the integration between variables, all hypotheses have been confirmed. In addition, in consistent with other previous studies, ERP has been found to have a positive and significant effect on organizational performance $(\beta=0.579, \mathrm{t}=15.293, \mathrm{p}<0.001)$ (Poston \& Grabski, 2001; Park, Suh, \& Yang, 2007). However, the effect of ERP on TQM and ERP on organizational excellence have not been linked before in the literature, it was found that they have positive and significant effect $(\beta=0.841, \mathrm{t}=44.301, \mathrm{p}<0.001)$ and $(\beta=0.634, \mathrm{t}=15.337, \mathrm{p}<0.001)$ respectively. In line with other previous studies, TQM was found to have a positive and significant effect at 0.001 level of significance $(\beta=0.605, \mathrm{t}=$ 12.973, $\mathrm{p}<0.001$ ) (Chong \& Rundun, 2004; Hassan \& Kerr, 2003). Moreover, TQM was found to have positive and significant effect on organizational excellence $(\beta=0.684, t=14.422, p<0.001)$, and organizational excellence has positive and significant effect on organizational performance $(\beta=0.825, \mathrm{t}=34.747, \mathrm{p}<0.001)$.

The mediation role of TQM and organizational excellence also were examined. TQM was found to have a partial mediation effect with 58\% influence of ERP on organizational performance at the 0.001 level of significance $(\beta=0.199, t=2.695, p<0.001)$. Similarly, organizational excellence with $81 \%$ influence of ERP on organizational performance, was found to have full mediation at the 0.001 level of significant $(\beta=0.491, t=12.332, p<0.001)$.

This study has many theoretical contributions. Besides examining the direct effect among variables due to the inconclusiveness finding in the previous literature, this study involves and examines new relationships between variables i.e. ERP-TQM and ERP-organizational excellence. In addition, this study fills the gap in the literature by examining the mediating effect of TQM and organizational excellence on the relationship between ERP and organizational performance. The results of this study will urge other researchers to investigate and examine other factors that may play some roles in these relationships. The framework of this study is a unique framework which suggested new relationships which never studied before.

In practice, the findings of this study have different practical implications. This study clears the way to managers and decision makers to involve TQM and excellence in their organizations while implementing ERP system. Due to the complexity of implementing ERP system, managers should think to have preliminary practice such as TQM to ease the ERP pre-implementation and post-implementation stages. Moreover, organizations face difficulties when implementing ERP system, therefore, other instruments needed to facilitate and pave the roads for successful implementation. Based on the conclusion of this study, managers should have some management practices in their organization such as TQM and excellence before thinking to implement ant ERP system. The reason behind that is to avoid the failure that may affect the entire organizations and may lead for collapsing. 
There are some limitations of this study. Similar to other previous research surveys, the data collected through self-reported which considered one of common method bias (Thornton, 2006). Cross-sectional as the research design of this study was used which considered another limitation. The biasness may also generate from the self-reported answers where the respondents translate their perceptions through the questionnaire. Therefore, future researches should include the mixed method design. In addition, longitudinal researches should be considered to test the effects of TQM, Excellence, and ERP on organizational performance in different point of times.

\section{References}

Abdinnour, S., \& Groen, E. (2009). Quality, ERP, and Performance at Hawker Beechcraft. Production and Inventory Management Journal, 45(1), 21-30.

Abebe, M. (2014). Electronic commerce adoption, entrepreneurial orientation and small-and medium-sized enterprise (SME) performance. Journal of Small Business and Enterprise Development, 21(1), 100-116. http://dx.doi.org/10.1108/JSBED-10-2013-0145

Adebanjo, D. (2001). TQM and business excellence: Is there really a conflict? Measuring Business Excellence, 5(3), 37-40. http://dx.doi.org/10.1108/13683040110403961

Anderson, M., \& Sohal, A. S. (1999). A study of the relationship between quality management practices and performance in small businesses. International Journal of Quality \& Reliability Management, 16(9), 859-877. http://dx.doi.org/10.1108/02656719910289168

Antony, J. P., \& Bhattacharyya, S. (2010). Measuring organizational performance and organizational excellence of SMEs-Part 2: An empirical study on SMEs in India. Measuring Business Excellence, 14(3), 42-52. http://dx.doi.org/10.1108/13683041011074209

Antony, J. P., \& Bhattacharyya, S. (2010). Measuring organizational performance and organizational excellence of SMEs-Part 1: A conceptual framework. Measuring Business Excellence, 14(2), 3-11. http://dx.doi.org/10. $1108 / 13683041011047812$

Arawati, A. (2005). The structural linkages between TQM, product quality performance, and business performance: Preliminary empirical study in electronics companies. Singapore Management Review, 27(1), $87-105$.

Attafar, A., Forouzan, B., \& Shojaei, M. (2012). Evaluation of Organizational Excellence Based on Peters and Waterman's Model in Tuka Steel Investment Holding. American Journal of Scientific Research, (50), 119-137.

Barker, T., \& Frolick, M. N. (2003). ERP implementation failure: A case study. Information Systems Management, 20(4), 43-49. http://dx.doi.org/10.1201/1078/43647.20.4.20030901/77292.7

Bendoly, E., \& Kaefer, F. (2004). Business Technology Complementarities: Impacts of the Presence and Strategic Timing of ERP on B2B E-commerce Technology Efficiencies. Omega, 32(5), 395-406. http://dx.doi.org/10.1016/j.omega.2004.02.004

Bhatt, G. D. (2000). An empirical examination of the effects of information systems integration on business process improvement. International Journal of Operations \& Production Management, 20(11), 1331-1359. http://dx.doi.org/10.1108/01443570010348280

Botta-Genoulaz, V., \& Millet, P. A. (2006). An investigation into the use of the ERP systems in the service sector. International Journal of Production Economics, 99, 202-221. http://dx.doi.org/10.1016/j.ijpe.2004.12.015

Brah, S. A., Tee, S. S. L., \& Rao, B. M. (2002). Relationship between TQM and performance of Singapore companies. International Journal of Quality \& Reliability Management, 19(4), 356-379. http://dx.doi.org/ $10.1108 / 02656710210421553$

Brah, S. A., Wong, J. L., \& Rao, B. M. (2000). TQM and business performance in the service sector: A Singapore study. International Journal of Operations \& Production Management, 20(11), 1293-1312. http://dx.doi.org/10.1108/01443570010348262

da Silveira, G. J., Snider, B., \& Balakrishnan, J. (2013). Compensation-based incentives, ERP and delivery performance Analysis from production and improvement perspectives. International Journal of Operation \& Production Management, 33(4), 415-441. http://dx.doi.org/10.1108/01443571311307307

Davenport, H. T. (2002). Putting the Enterprise into the Enterprise System. Harvard Business Review, 9(3), 14.

Demirbag, M., Koh, S. C. L., Tatoglu, E., \& Zaim, S. (2006). TQM and market orientation's impact on SMEs' 
performance. Industrial Management \& Data System, 106(8), 1206-1228. http://dx.doi.org/10.1108/ 02635570610710836

Dooyoung, S., Kalinowski, J. G., \& El-Enein, G. (1998). Critical implementation issues in total quality management. SAM Advanced Management Journal, 63(1), 10-14.

Ehigie, B. O., \& McAndrew, E. B. (2005). Innovation, diffusion, and adoption of total quality management (TQM). Management Decision, 43(6), 925-940. http://dx.doi.org/10.1108/00251740510603646

Everdingen, Y. V., Hillegersberg, J. V., \& Waarts, E. (2000). ERP Adoption by European midsize companies. Communication of the ACM, 43(3), 27-31. http://dx.doi.org/10.1145/332051.332064

Fang, M., \& Lin, F. (2006). Measuring the Performance of ERP System-from the Balanced Scorecard Perspectives. Journal of American Academy of Business, 10(1), 256-263.

Feng, J., Prajogo, D. I., Tan, K. C., \& Sohal, A. S. (2006). The impact of TQM practices on performance: A comparative study between Australian and Singaporean organizations. European Journal of Innovation Management, 9(3), 269-278. http://dx.doi.org/10.1108/14601060610678149

Fotopoulos, C. V., \& Psomas, E. L. (2010). The structural relationships between TQM factors and organizational performance. The TQM Journal, 22(5), 539-552. http://dx.doi.org/10.1108/17542731011072874

Gadenne, D., \& Sharma, B. (2009). An investigation of the hard and soft quality management factors of Australian SMEs and their association with firm performance. International Journal of Quality \& Reliability Management, 26(9), 865-880. http://dx.doi.org/10.1108/02656710910995064

Hackman, J., \& Wageman, R. (1995). Total Quality Management: Empirical, conceptual, and practical issues. Administrative Science Quarterly, 40, 309-342. http://dx.doi.org/10.2307/2393640

Hair, J. F., Anderson, R. E., Tatham, R. L., \& Black, W. C. (2010). Multivariate Data.

Hair, J. F., Hult, G. T., Ringle, C. M., \& Sarstedt, M. (2014). A primer on partial least squares structural equation modeling (PLS-SEM). Sage.

Hasan, M., \& Kerr, R. M. (2003). The relationship between total quality management practices and organizational performance in service organizations. The TQM Magazine, 15(4), 286-291. http://dx.doi.org/ $10.1108 / 09544780310486191$

HassabElnaby, H. R., Hwang, W., \& Vonderembse, M. A. (2012). The impact of ERP implementation on organizational capabilities and firm performance. Benchmarking, 19(4/5), 618-633. http://dx.doi.org/10.1 $108 / 14635771211258043$

Hayes, D. C., Hunton, J. E., \& Reck, J. L. (2001). Market reaction to ERP implementation announcements. Journal of Information System, 15(1), 3-18. http://dx.doi.org/10.2308/jis.2001.15.1.3

Hendricks, K. B., \& Singhal, V. R. (1997). Does implementing an effective TQM program actually improve operating performance empirical evidence from firms that have Won Quality Awards? Management Science, 43(9), 1258-1274. http://dx.doi.org/10.1287/mnsc.43.9.1258

Hitt, L. M., \& Brynjolfsson, E. (1996). Productivity, business profitability, and consumer surplus: Three different measures of information technology value. MIS Q, 20(2), 121-142. http://dx.doi.org/10.2307/ 249475

Hwang, W., \& Min, H. (2013). Assessing the impact of ERP on supplier performance. Industrial Management \& Data Systems, 113(7), 1025-1047. http://dx.doi.org/10.1108/IMDS-01-2013-0035

Ionica, A., \& Baleanu, V. (2010). TQM and business excellence. Annals of the University of Petroşani, Economics, 10(4), 125-134.

Jha, V. S., \& Joshi, H. (2007). Relevance of Total Quality Management (TQM) or business Excellence Strategy Implementation for Enterprise Resource Planning (ERP)-A Conceptual Study.

Kang, S., Park, J. H., \& Yang, H. D. (2008). ERP alignment for positive business performance : Evidence from Korea's ERP market. Journal of Computer Information Systems, 25-38.

Kanji, G. (2002). Measuring Business Excellence. London: Routledge.

Kaplan, R. S., \& Norton, D. P. (1992). The balanced scorecard \pm measures that drive Performance. Harvard Business Review, 70-79.

Kaplan, R. S., \& Norton, D. P. (2000). Having trouble with your strategy? Then map it. Harvard Business Review, 78(5), 167-176. 
Kerlinger, F. N., \& Lee, H. B. (2000). Foundations of behavioral research (4th ed.). Harcourt College Publishers: Orlando, US.

Kumar, V., Maheshwari, B., \& Kumar, U. (2002). Enterprise Resource Planning Systems Adoption Process: A Survey of Canadian Organizations. International Journal of Production Research, 40, 509-523. http://dx.doi. org $/ 10.1080 / 00207540110092414$

Laframboise, K. (2002). Business Performance and Enterprise Resource Planning. Proceedings of ECIS 2002 Conference, Gdansk, Poland.

Laframboise, K., \& Reyes, F. (2005). Gaining competitive advantage from integrating enterprise resource planning and total quality management. Journal of Supply Chain Management, 41(3), 49-64. http://dx.doi. org/10.1111/j.1055-6001.2005.04103005.x

Lee, C. Y. (2004). TQM in small manufacturers: An exploratory study in China. International Journal of Quality \& Reliability Management, 21(2), 175-197. http://dx.doi.org/10.1108/02656710410516970

Lee, P. M. (2002). Sustaining business excellence through a framework of best practices in TQM. The TQM Magazine, 14(3), 142-149. http://dx.doi.org/10.1108/09544780210425883

Lyons, K., Acsente, D., \& Waesberghe, M. V. (2008). Integrating knowledge management and quality management to sustain knowledge enabled excellence in performance. The journal of information and knowledge management systems, 38(2), 241-253.

Manetti, J. (2001). How Technology Is Transforming Manufacturing. Production and Inventory Management Journal, 42(1), 54-64.

Marc, S. J., \& Gyu, K. C. (2003). Implementing Enterprise Resource Planning systems with Total Quality Control and Business Process Reengineering. International Journal of Operations \& Production Management, 23(4), 418-429. http://dx.doi.org/10.1108/01443570310467339

Markus, M. L., Axline, S., Petrie, D., \& Tanis, C. (2000). Learning from adopters' experiences with ERP: Problems encountered and success achieved. Journal of Information Technology, 15(4), 245-266. http://dx.doi.org/10.1080/02683960010008944

Masli, A., Richardson, V. J., Sanchez, J. M., \& Smith, R. E. (2010). Return to IT excellence: Evidence from financial performance around information technology excellence awards. International Journal of Accounting Information System, 12, 189-205. http://dx.doi.org/10.1016/j.accinf.2010.10.001

Mele, C., \& Colurcio, M. (2006). The evolving path of TQM: Towards business excellence and stakeholder value. International Journal of Quality \& Reliability Management, 23(5), 464-489. http://dx.doi.org/10. $1108 / 02656710610664569$

Moghadami, S. (2005). Mysteries of organizational excellence. Tadbir Monthly Magazine, 144.

Nor Hazilah, A. M. (2004). Quality management in the public sector: An empirical survey of the Ministry of Health Hospitals in Peninsular Malaysia. Unpublished PhD dissertation: Universiti Malay.

Oakland, J. S. (1995). Total Quality Management (Oxford: Butterworth-Heinemann).

Pacheco-Comer, A. A., \& González-Castolo, J. C. (2012). An empirical study in selecting Enterprise Resource Planning Systems: The relation between some of the variables involve on it. Size and Investment, Procedia Technology, 3, 292-303. http://dx.doi.org/10.1016/j.protcy.2012.03.032

Park, J. H., Suh, H. J., \& Yang, H. D. (2007). Perceived absorptive capacity of individual users in performance of Enterprise Resource Planning (ERP) usage: The case for Korean firms. Information \& Management, 44(1), 300-312. http://dx.doi.org/10.1016/j.im.2007.02.001

Peffers, K., \& Dos Santos, B. L. (1996). Performance effects of innovative IT applications over time. IEEE Trans Eng Manage, 43(4), 381-392. http://dx.doi.org/10.1109/17.543980

Pinar, M., \& Girard, T. (2008). Investigating the Impact of Organizational Excellence and Leadership on Business Performance: An Exploratory Study of Turkish Firms. SAM Advanced Management Journal, 73(1), 29-45.

Poston, R., \& Grabski, S. (2001). Financial impacts of enterprise resource planning implementations. International Journal of Accounting Systems, 2(4), 271-294. http://dx.doi.org/10.1016/S1467-0895(01) 00024-0

Prajogo, D. I., \& Sohal, A. S. (2004). The multidimensionality of TQM practices in determining quality and innovation performance-an empirical examination. Technovation, 24(6), 443-453. http://dx.doi.org/10.1016/ 
S0166-4972(02)00122-0

Rao, S. S. (2000). Enterprise resource planning: Business needs and technologies. Industrial Management \& Data Systems, 100(2), 81-86. http://dx.doi.org/10.1108/02635570010286078

Rashid, K., \& Aslam, H. (2012). Business excellence through total supply chain quality management. Asian Journal on Quality, 13(3), 309-324. http://dx.doi.org/10.1108/15982681211287829

Salaheldin, S. I. (2009). Critical success factors for TQM implementation and their impact on performance of SMEs. International Journal of Productivity and Performance Management, 58(3), 215-237. http://dx.doi. org/10.1108/17410400910938832

Shang, S., \& Seddon, P. B. (2000). A Comprehensive framework for classifying the benefits of ERP systems. Proceedings of the Sixth Americas Conference on Information Systems, Long Beach, Ca, August 10-13.

Sharma, M., \& Kodali, R. (2008). TQM implementation elements for manufacturing excellence. The TQM Magazine, 20(6), 599-621. http://dx.doi.org/10.1108/17542730810909365

Sila, I., \& Ebrahimpour, M. (2002). An investigation of the total quality management survey based research published between 1989 and 2000: A literature review. International journal of Quality and Reliability Management, 19(7), 902-970.

Sohail, M. S., \& Hoong, T. B. (2003). TQM practices and organizational performances of SMEs in Malaysia: Some empirical observations. Benchmarking, 10(1), 37-53. http://dx.doi.org/10.1108/14635770310457539

Sohal, A. S., \& Terziovski, M. (2000). TQM in Australian manufacturing: Factors critical to success. International Journal of Quality \& Reliability Management, 17(2), 158-167. http://dx.doi.org/10.1108/ 02656710010304564

Stratman, J. K., \& Roth, A. V. (2002). Enterprise resource planning (ERP) competence constructs: Two-stage multi-item scale development and validation. Decision Sciences, 33(4), 601-628. http://dx.doi.org/10.1111/j. 1540-5915.2002.tb01658.x

Terziovski, M., \& Samson, D. (1999). The link between total quality management practices and organizational performance. The International Journal of Quality \& Reliability Management, 16(3), 226. http://dx.doi.org/ $10.1108 / 02656719910223728$

Thornton, G. C. (2006). The relationship between supervisory and self-appraisals of executive performance. Personnel Psychology, 21(4), 441-455. http://dx.doi.org/10.1111/j.1744-6570.1968.tb02044.x

Uwizeyemungu, S., \& Raymond, L. (2010). Linking the Effects of ERP to Organizational Performance: Development and Initial Validation of an Evaluation Method. Information Systems Management, 27(1), 25-41. http://dx.doi.org/10.1080/10580530903455122

Velcu, O. (2007). Exploring the effects of ERP systems on organizational performance: Evidence from Finnish companies. Industrial Management \& Data Systems, 107(9), 1316-1334. http://dx.doi.org/10.1108/0263 5570710833983

Wang, H., \& Yen, Y. (2012). An empirical exploration of corporate entrepreneurial orientation and performance in Taiwanese SMEs: A perspective of multidimensional construct. Total Quality Management, 23(9), 1035-1044. http://dx.doi.org/10.1080/14783363.2012.670917

Weill, P. (1992). The Relationship Between Investment in Information Technology and Firm Performance: A Study of the Valve Manufacturing Sector. Information Systems Research, 3(4), 307-333. http://dx.doi.org/ 10.1287/isre.3.4.307

Wier, B., Hunton, J., \& HassabElnaby, H. R. (2007). Enterprise resource planning systems and non-financial performance incentives: The joint impact on corporate performance. International Journal of Accounting Information Systems, 8(3), 165-190. http://dx.doi.org/10.1016/j.accinf.2007.05.001.

Yasin, M. M., Kunt, J. A. M., \& Zimmerer, T. W. (2004). TQM practices in service organizations: An exploratory study into the implementation, outcomes and effectiveness. Managing Service Quality, 14(5), 377-389. http://dx.doi.org/10.1108/09604520410557985.

\section{Copyrights}

Copyright for this article is retained by the author(s), with first publication rights granted to the journal.

This is an open-access article distributed under the terms and conditions of the Creative Commons Attribution license (http://creativecommons.org/licenses/by/3.0/). 